\title{
Abstracts \\ www.anatomy.org.tr \\ doi:10.2399/ana.12.113s \\ Abstracts for the 14th National Congress of Anatomy 28 June-1 July 2012, Ankara, Turkey
}

\section{Invited Lectures}

\author{
$(\mathrm{I}-1-\mathrm{I}-2)$
}

I-1

\section{Arcade of Struthers: a controversial anatomical entity Akkın SM \\ Department of Anatomy, Cerrahpaşa Faculty of Medicine, istanbul University, Istanbul, Turkey \\ sma@deomed.com}

The term "arcade of Struthers", which is used routinely in upper extremity surgery, indicates complex fascial, fibrous and musculofibrous structures that show a very close neighborhood with the ulnar nerve and accompanying superior collateral ulnar artery at the anteroposterior compartmental transition level of the nerve on the medial intermuscular septum of arm. Struthers pointed out in his original publication in 1854 that the ulnar nerve had a close relation to a ligamentous cord arising from the coracobrachialis tendon at this level in some cases. He referred to the term "internal brachial ligament" as a means of distinguishing this cord from the true septum. The term "arcade", however, was attributed to Struthers by Kane et al. in 1973. The main clinical significance of this structure, which is known as one of the five potential entrapment sites around the elbow, is the recurrent traumatic neuritis because of the potential kinking if anterior transposition of ulnar nerve is performed, as mentioned by Spinner and Kaplan in 1976. There is a controversy in the literature regarding the terminology, description and clinical importance of the arcade of Struthers. The results of the anatomical studies on this structure showed significant differences and ranged from $0 \%$ to $100 \%$. Almost only consensus regarding this arcade, that shows individual variations, is that it is localized approximately $8 \mathrm{~cm}$ proximal to the medial epicondyle. In this paper, this controversial anatomic entity is revisited in company with the preliminary results on our both fixed and fresh cadaver studies.

Keywords: Arcade of Struthers, internal brachial ligament, medial intermuscular septum of arm, ulnar nerve

\section{I-2}

\section{Mechanics, biomechanics and mathematical modelling}

Tümer ST

Middle East Technical University, Northern Cyprus Campus, Güzelyurt, KKTC

tumer@metu.edu.tr

Classical mechanics deals with the behavior of materials under the action of forces. It is the most ancient branch of physics that started with construction and was essential in machinery during industrial revolution. Although analysis of biological systems with mechanics principles dates back to $400 \mathrm{BC}$, biomechanics is relatively recent in research and education. Biomechanics evolved within few decades staring with experimental studies continued with mathematical modeling with the evolution of digital computers. In this presentation the basic principles of mechanics and their biomechanical implementations with their limitations will be discussed. Knee and shoulder joint models, gait, modeling weight lifting activities, developed in the department of Mechanical Engineering METU will be discussed. 


\title{
Satellite Symposiums
}

\author{
(C-8 - C-11)
}

\section{C-8}

\section{A cadaver study and MRI analyses to show in vivo occurrence of myofascial force transmission in human muscles}

Yücesoy $\mathrm{CA}^{*}$, Yaman A*, Öztürk $\mathrm{C}^{*}$, Huijing $\mathrm{PA}^{* *}$

*Institute of Biomedical Engineering, Bogaziçi University, İstanbul, Turkey; **Human Movement Science Faculty, Vrije University, Amsterdam, Netherlands

Evidence in situ show feasibility and effects of transmission of muscle force via non-myotendinous pathways to muscular and non-muscular structures. However, in vivo occurrence of such epimuscular myofascial force transmission (EMFT) has not been proved. Our goal was to test the hypotheses: (1) Global strain imposed on the $\mathrm{m}$. gastrocnemius by knee joint movement and local strains within the muscle are quite different. (2) EMFT does occur in vivo among synergistic and antagonistic human muscles. Cadaver study: both heads of $\mathrm{m}$. gastrocnemius $(n=7)$ were transected at the mid-belly. After imposed knee angle changes, gap lengths between transected ends were quantified. Global strain was calculated as gap length change/segment length. MRI study: Subjects $(n=5)$ were positioned prone with the ankle fixed at $90^{\circ}$. The knee angle in the undeformed and deformed states equaled $173^{\circ} \pm 3^{\circ}$ and $150^{\circ} \pm$ $6^{\circ}$. MR image sets were registered to calculate local strains. Both hypotheses are confirmed: after knee movement without ankle movement, global strains imposed on m. gastrocnemius remained much less (approximating 1.5\%) than the local strains and local lengthening within the muscle was shown to occur simultaneously with local shortening (maximally by $+34.2 \%$ and $-32.6 \%$, respectively) at different locations. Moreover, for the synergistic $\mathrm{m}$. soleus and deep flexors, as well as for antagonistic anterior crural and peroneal muscles, also sizable local deformations were found (minimum peak strain equaled $23.3 \%$ ) despite the global isometric condition. These findings indicate that, also in vivo, muscles are not mechanically independent.

\section{C-9}

Mechanical modeling of soft biological tissues by fractional calculus

Demirci N, Tönük E

Middle East Technical University, Department of Mechanical Engineering, Ankara, Turkey

It is known for long that soft biological tissues exhibit both solid-like and fluid-like behavior and many of the mechanical models for soft tissues include combination of solid and fluid elements to model this behavior. In this work, in addition to elastic solid, using fractional calculus, an intermediate material which can continuously vary from solid-like to fluid-like behavior is used. It is also shown that fractional calculus modeling is not just a mathematical abstraction but can model fractal structure seen in the anatomy of many soft biological tissues.

\section{C-10}

\section{Comparison of hip joint center estimation methods with anatomical hip joint center}

\section{Yousefi A}

Department of Mechanical Engineering, Middle East Technical University, Ankara, Turkey

Gait analysis is a tool for diagnosis, rehabilitation planning and evaluation for medical doctors. During mathematical modeling in gait analysis, data like masses, mass centers and mass moments of inertias of body segments and centers of joints are needed. If required, these data may be obtained precisely however it requires much time and effort, therefore, mostly in practice estimated with certain accuracy. Hip joint center estimation, among these data, received attention in the literature. In this work some of the widely used hip joint center estimation methods will be compared with anatomical hip joint center for healthy subjects and subjects with certain hip pathologies.

\section{C-11}

\section{Biomechanical modeling of human movement}

Aritan S

Hacettepe University, School of Sports Science and Technology, Biomechanics Research Group, Ankara, Turkey

Biomechanics suffers from one very serious limitation; in general it is impossible to measure forces inside the human body for technical and ethical reasons. In order to measure the force inside a human body requires an operation to implement a force transducer. In addition to technical complications and calibration problems of force transducer, the subject would also be at risk of surgical infections. Therefore, modeling in biomechanics works as an interface between the body and measurement settings. Developing a biomechanical model itself improves the understanding of the mechanical system's dynamics and the structure. Basically, there are two types of approaches in biomechanical modeling. The first one is inverse dynamics and the 
second one is forward dynamics or direct dynamics. Inverse dynamics calculation is used to determine joint forces and torques based on a time history of displacements from experimental kinematic data, including velocities and accelerations. Mass and inertial characteristics of segments are also required in this method. In a forward dynamical analysis the joint torques are the inputs and the body motion is the output. It is critical to understand what generate this joint torques. Muscles are the actuator in this method. Therefore the correct input into the model is definitely neural input, which drives the muscles. Whichever approach is used for modeling, first of all, the equation of motion has to be derived. The dynamics of biomechan- ical systems is based on classical mechanics. The simplest element of a multi-body biomechanical system is a free particle which can be treated by Newton's equations. The rigid body that is a key element in the modeling was introduced by Euler. Thus the equations obtained are known in human-body dynamics as Newton-Euler equations. Modeling is widely used in all biomechanics from kinematics to dynamics. In fact, advanced biomechanical modeling requires sophisticated simulation tools which can model accurately enough the physical world at sufficient speed and allow user interaction.

Keywords: Biomechanics, modeling, simulation, human movement, dynamics 


\title{
Oral Presentations
}

\author{
(0-1 - 0-26)
}

\section{0-1}

\section{Three master founders of the Turkish Anatomy: Mazhar Pasha, Nurettin Ali Berkol and Zeki Zeren}

Mesut R

Department of Anatomy, Faculty of Medicine, Trakya University, Edirne, Turkey

It can be named as the "golden century of anatomy" (between 1875 and 1973) in which the anatomy has founded and developed by becoming an independent lesson with the progression of modern anatomy education. The three masters Hasan Mazhar Pasha (1845-1920), Nurettin Ali Berkol (1881-1955) and Zeki Zeren (1900-1973) who left their mark on this period were in a relation of both teacher-students and predecessorsuccessor. This "trio" can be compared with the "Socrates, Plato and Aristotle" trio of the world philosophy history or "Hippocrates, Galen and Avicenna" trio of the history of world medicine. Both three of them were born and died in Istanbul. They all have expertise in anatomy in France, promoted the French Anatomy ecole in our country and represented the Turkish anatomy in foreign countries. They have devoted all their academic life to anatomy, raised young anatomists by organizing a team and published Turkish textbooks. They have provided Turkish anatomical terms through their courses and textbooks. They have witnessed the last years of the Ottoman Empire and the first years of the Republic of Turkey, and they have served the country by raising medical doctors despite the wars, depressions, revolutions, shortage and poverty.

Keywords: History of Turkish anatomy, Mazhar Pasha, Nurettin Ali Berkol, Zeki Zeren

\section{0-2}

From mythology to anatomy: a place hiding for mythologic stories

Durgun B

Department of Anatomy, Faculty of Medicine, Çukurova University, Adana, Turkey

In this study, the reflection of mythology to the medical and anatomical terminology is presented with the stories behind the words. There is a story behind each medical and anatomical term. Medical terminology conserves these stories as Pandora's Box. The term mythology comes from the Greek mythos means that a traditional story explaining a natural or social phenomenon, and typically involving supernatural beings or events", and from logos meaning "word" or "reason". Mythology is a creation of human consciousness that attempt to interpret events and observations that are beyond the scope of systematic scientific discourse. Both myths and science offer explanations of the cosmos. A key difference is that information about the universe presented in myths is not testable, whereas science is designed to be tested repeatedly. Mitologia, especially Greek mythology, have surpassed boundaries of time and spaces. There has been a direct relationship between medical and anatomical terminology and mythology. This has meant that ancient practitioners essentially named medical conditions using words that were sometimes inextricably linked to mythology. Even today some medical terminology can still be understood from its mythological root alone. This relationship applies to the entirety of medicine from anatomy to clinical signs.

Keywords: Medical terminology, anatomy, mythology

\section{0-3}

The place of clinical anatomy unit in postgraduate and continuing medical education

Sindel $\mathrm{M}^{*}$, Oğuz N*, Arıcan RY**, Özsoy U*, Demirel B**, Şenol $\mathrm{Y}^{* * * *}$

*Department of Anatomy, Faculty of Medicine, Akdeniz University, Antalya, Turkey; ${ }^{*}$ Department of Anatomy, Faculty of Medicine, Near East University, Nicosia, Turkish Rebuplic of North Cyprus; ***Department of Anatomy, Faculty of Medicine, Ordu University, Ordu, Turkey; $* * * *$ Department, of Medical Education, Faculty of Medicine, Akdeniz University, Antalya, Turkey

Anatomy is one of the most basic courses of medical education and is also required for good medical practices. The concept of clinical anatomy has become widely used since the late 1970s. Clinical anatomy is associated with the study of clinical applications of human anatomy and it focuses on special tissues and structures. Many physicians, especially surgeons, in the process of gaining new experiences related to their areas, benefit from clinical anatomy. The first studies about clinical anatomy in our faculty began in 2000. In 2006, these studies were collected under clinical anatomy teaching and research unit. The purpose of this paper is to introduce the first established clinical training and research unit in our country, share experiences and present opinions about the unit. AUMF Clinical anatomy teaching and research unit was established in 2006 for the continuity of national and international symposia and courses and in order to contribute to scientific studies. The unit organized more than 40 courses between 2007-11. Post-graduate students as well as specialists in the process of continuing medical education participated the courses. Knee, shoulder, hip arthroscopy courses, peripheral nerve dissection, ear surgery are some of the organized courses. Feedback was taken after courses. The results of the feedbacks given after the courses were evaluated. Clinical Anatomy Education and Research unit 
which is the first in our country is an important value for undergraduate, postgraduate and continuing medical education. The unit allows multidisciplinary studies.

Keywords: Clinical anatomy, education, surgical training

\section{0-4}

\section{Use of touchscreen virtual education panels in anatomy labs}

Yazar F, Develi S, Yalçın B

Department of Anatomy, Gülhane Military Medical Academy, Ankara, Turkey

Providing new training materials to educational environment and using newly developed computer-assisted medical applications has become a necessity. Today virtual computer-aided programs have been widely used in methods of diagnosis and treatment. Therefore, many educational materials supporting these methods are used as a basic training material in courses such as anatomy education. Besides making anatomy training more effective and efficient, using virtual reality medical education materials in medical practice from the beginning will provide easy adaptation for newly developed products such as laparascopy, robotic surgery. In anatomy training, interactive educational applications, virtual cadavers, animations, videos and other visual materials will be used effectively by students and lecturers and will make learning easier and more attractive. The touch screen virtual education panels, will facilitate the learning of students. Besides being a resource material in the dissection hall for students and lecturers, these panels will make easy to learn and understand anatomic structures by presenting virtual cadavers, animations, radiological images, atlases at the same time.

Keywords: Virtual panel, anatomy education

\section{0-5}

\section{Names which originate from plants within Terminologia anatomica}

Unur E, Ertekin T, Acer N, Çınar S, Özçelik O

Department of Anatomy, Faculty of Medicine, Erciyes University Kayseri, Turkey

Our aim is to identify the names which originate from plants and to determine how often they got within Terminologia anatomica (TA). The names of vegetable origin were determined by scanning TA was published in 1998 by the Federative Committee on Anatomical Terminology. Turkish meanings of these names were found in various dictionaries. We have determined 7537 anatomical terms in TA which defined the structures of human body. Total 25 plants name were found in all 7537 names. These names divided in two groups. Whether the first group names (13 names) were reminded directly by plant, the second group names (12 names) were not reminded directly by plant names but their origins were based on plant origin. We examined how often these names are repeated in TA. We determined that these names repeated in 647 times. The ratio of names which based on plant origin was $7.4 \%$ in total terms of TA. The anatomy is the first step in medical education. Education of terminology is the base of anatomy lesson. The science of anatomy possesses very rich terminological knowledge. The basis of this terminology is based on words which originate from the Latin and Greek. If we determine these plant origin names and explain them to student with knowing the meaning of these names, we may contribute the understanding of anatomy easier.

Keywords: Terminologia Anatomica, terminologia, medical education

\section{0-6}

\section{A detailed study of the relationship between the external branch of the superior laryngeal nerve and superior thyroid artery}

Yalçın B*, Develi S*, Tubbs RS**

*Department of Anatomy, Gülhane Military Medical Academy, Ankara, Turkey; ${ }^{* *}$ Department of Pediatric Neurosurgery, Children's Hospital, Birmingham, Alabama, USA

The external branch of the superior laryngeal nerve may be at risk when a surgeon is dissecting to ligate the superior thyroid artery during thyroid surgery. The relation of the nerve to the artery is extremely variable. Relationships between them were aimed to investigate in detail. In 60 larynx specimens taken from human cadavers, 81 nerves and arteries with their branches were carefully dissected. Position of the nerve was classified; medial (group I, in $76.5 \%$ sides), lateral (group II, in $20.9 \%$ sides) or posterior (group III, in $2.4 \%$ sides) to the origin point of the STA. Group Ia: In a part of group I (in $39.5 \%$ sides), the nerve was located medial to the origin point of the artery and passed downward on the pharynx and parallel to the artery. Group Ib: the nerve was also located medial to the origin point of the artery. But differently, it crossed it or its branches (in 37\% sides). Group II: The nerve was located lateral to the origin point of the artery, and crossed it or its branches. Group III: The nerve coursed downward posterior to the artery, and then pierced the pharynx above and below the upper pole of the thyroid gland. The topography of the nerve showed much more variability in its relationship to the artery. So, the relationships of both structures with their branches should be kept in mind and be careful during surgery.

Key words: Superior laryngeal nerve; external laryngeal nerve; superior laryngeal artery; thyroid gland

\section{0-7}

The origin, course and branching pattern of the pudendal nerve in Turkish adult cadavers

Gürses IA* ${ }^{*}$, Gayretli Ö*, Kocabıyık N**, Kale A*, Coşkun O*, Öztürk A*

*Department of Anatomy, Faculty of Medicine, Istanbul University, Istanbul, Turkey; **Department of Anatomy, Gülhane Military Medical Academy, Ankara, Turkey

The pudendal nerve (S2-S4), which is a branch of the sacral plexus, is a motor and sensory nerve of the perineum. While per- 
S118 14th National Congress of Anatomy, 28 June-1 July 2012, Ankara, Turkey

forming procedures like nerve anastomosis, neurolysis or nerve block for chronic perineal pain syndrome or even ischial osteotomy, a sound knowledge of its anatomy is needed. The origin, course and branching pattern of the pudendal nerve regarding its relation with the sacrospinous ligament and the pudendal vessels were investigated in 10 cadavers. The length between its origin and entry point to the Alcock's canal and the distance of it to the ischial spine were measured with a digital caliper. The nerve originated from S2-S3 (75\%) on 15 sides; S2 to S4 (10\%) on 2 sides; S3-S4 (5\%), S1 to S3 (\%5) and S3 to S5 (5\%) on 1 side each respectively. The mean length of the nerve was $39,2 \mathrm{~mm}$ $(27,3-51,6 \mathrm{~mm})$. The nerve passed lateral to the ischial spine on 16 sides and medial on 4 sides. The mean distances were $2,9 \mathrm{~mm}$ $(0,8-4,4 \mathrm{~mm})$ and $2,4 \mathrm{~mm}(1,05-3,4 \mathrm{~mm})$ respectively. The nerve was classified into 3 types regarding its branching pattern and the relation of its branches to the sacrospinous ligament. Type I was defined as a single trunk (10\%), Type II as double trunk $(60 \%)$ and Type III as triple trunk $(30 \%)$. This study outlines the topographic anatomy of the pudendal nerve and can be of importance for related procedures.

Keywords: Pudendal nerve, sacrospinous ligament, branching pattern

\section{0-8}

Bilateral persistent sciatic vein: report of a case with developmental, histological and clinical aspects

Koç $T^{*}$, Gilan IY**, Külekçi GD**, Kurtoğlu $Z^{*}$

*Department of Anatomy, Faculty of Medicine, Mersin University, Mersin, Turkey; **Department of Histology, Faculty of Medicine, Mersin University, Mersin, Turkey

Bilateral large variant vein was encountered in the lower extremity. It was aimed to identify the structural characteristics of this rare case and then, regarding the structural features, to overview its formation process and denomination. During the routine dissection of a 93-year-old male cadaver, bilateral large variant veins were found at the thigh. Valves of the veins were examined and evaluated together with the vascular wall histology. The variant vein was loosely attached to the sciatic nerve by fibrous tissue and made anastomoses with the popliteal vein in the popliteal fossa on each side. The popliteal vein was hypoplastic on both sides. The right variant vein was passing among the fibers of the adductor magnus muscle $56.2 \mathrm{~mm}$ above the adductor hiatus, which is corresponding with third perforating branch of deep femoral vein. The left one was turning to the anterior part of thigh around the trochanter minor, which is corresponding with descending branch of the medial circumflex femoral vein. Both variant veins had three well-developed and one incomplete valves. In accordance with the findings, the variant vein was concluded to be an embryonic remnant, rather than an acquired one subsequent to any obstruction of the femoral vein. It was denominated as "persistent sciatic vein". Such a variation would be important with respect to the risk of complication during popliteal sciatic nerve blockade.

Keywords: Sciatic vein, deep femoral vein, popliteal vein, variation, sciatic nerve.

\section{0-9}

Neuroprotective role of melatonin on recovery after sciatic nerve injury: a combined study using functional, electrophysiological, biochemical and electron-microscopic analysis

$\underline{\text { Kaya } Y^{*}}$, Sarıkcıŏglu L *, Aslan $M^{* *}$, Kencebay C ***, Demir $\mathrm{N}^{* * * *, * * * *}$, Derin $\mathrm{N}^{* * *}$, Angelov $\mathrm{DN}^{* * * * *}$, Yildırım $\mathrm{FB}^{*}$

*Department of Anatomy, Faculty of Medicine, Akdeniz University, Antalya, Turkey; **Department of Biochemistry, Faculty of Medicine, Akdeniz University, Antalya, Turkey; ***Department of Biophysics, Faculty of Medicine, Akdeniz University, Antalya, Turkey; ****Department of Histology and Embryology, Faculty of Medicine, Akdeniz University, Antalya, Turkey; $* * * *$ Electron Microscopy Unit, Faculty of Medicine, Akdeniz University, Antalya, Turkey*****; University of Cologne, Anatomy Institute I, Cologne, Germany

Following tissue injury melatonin is known to reduce detrimental effects of free radicals by stimulating superoxide dismutase, glutathione peroxidase, and glutathione reductase. Neuroprotective effects after peripheral nerve injury have been suggested, but not studied in details. Therefore, we aimed to elucidate effects of melatonin on the recovery of the lesioned rat sciatic nerve by means of a combined analysis including functional, electrophysiological, biochemical and electron-microscopic approaches. A total number of 90 rats were randomly distributed into 6 groups: control (Group1), sham-operated (Group 2), sciatic nerve transection (Group 3), sciatic nerve transection + melatonin treatment (Group 4), sciatic nerve crush (Group 5), sciatic nerve crush + melatonin treatment (Group 6). Melatonin was administered intraperitoneally at dose of $50 \mathrm{mg} / \mathrm{kg} /$ day for six weeks (42 days). Evaluation of recovery was analyzed by assessment of the sciatic functional index based on walking track analysis, somatosensory evoked potentials, and biochemical quantification of malondialdehyde, superoxide dismutase, catalase, glutathione peroxidase and ultrastructural analysis. Our data show beneficial effect of melatonin on sciatic nerve recovery from functional, electrophysiological, biochemical and ultrastructural aspects. Rats treated with melatonin demonstrated better structural preservation of the myelin sheaths compared to the non-treated group. The biochemical analysis confirmed the neuroprotective effects of melatonin displaying lower lipid peroxidation, and higher SOD-, CAT-, GPx- activities in comparison to non-treated groups. The beneficial effects of melatonin administration on the recovery of the injured sciatic nerve may be attributed to its antioxidant properties.

Keywords: Melatonin, antioxidant enzymes, nerve injury, combine analysis

\section{0-10}

\section{Importance of the cross sectional anatomy in medical education}

Yalçın B

Department of Anatomy, Gülhane Military Medical Academy, Ankara, Turkey

The study of cross-sectional anatomy serves two purposes; 1) as a visual medium to facilitate an understanding of the structural 
organization of the human body and 2) as preparation for the study of tomographic (two-dimensional) slice images, such as CT and MR. It is important to appreciate that one need not desire to be a radiologist in order to benefit from a study of cross-sectional anatomy. A study of human anatomy from cross-sectioned cadaveric specimens provides a visually unique representation of the positions, sizes, shapes, and relationships of structures; this in turn facilitates an appreciation for the three-dimensional organization of the body. During the medical education, whirlwind rotations through the various clinical departments of the teaching hospital, the students are constantly surrounded by plain-film x-ray, CT, MR, and ultrasound images of the internal anatomy; however, clinical training demands leave little time to review the basic anatomy that would lend to interpreting these images. In retrospect, the medical student realizes that the best time to have learned to identify anatomical structure in tomographic images was during gross anatomy. Because of that, cross-sectional anatomy should be put into curriculum and taught with gross anatomy in first or second class of medical faculty, even in other medical schools. Additionally, high-quality cadaveric cross-sectional and radiographic images should be put into the examinations to make progress in this area.

Keywords: Cross-sectional anatomy, medical education

\section{0-11}

The development of anatomy education and contributions of military medical doctors in Turkey

Kocabıyık N*, Uçar $M^{* *}$, Yıldız $S^{*}$, Yazar F*

*Department of Anatomy, Gülhane Military Medical Academy, Ankara, Turkey; ${ }^{*}$ Department of Medical History and Deontology, Gülhane Military Medical Academy, Ankara, Turkey

The exact date 14th March 1827 when Tibhane-i Amire was founded to give modern medical education might also be considered as the starting point of modern anatomy education in the country. Anatomy classes had been delivered by Muneccim Osman Efendi. Six different books were used in the Anatomy classes and illustraions were used instead of cadavers. Name of the school was changed as Mekteb-i Tibbiye-i Adliye-i Sahane and Prof.Bernard from Vien was assigned as dean. From archives dating back to 1849 , it is understood that anatomy and autopsy classes were taught in the name "Professional Anatomy and Observing Cadaver" to military cadets and medical military cadets in the 6th and 7th grades by Dr. Vardihler. In 1866, we understand that the anatomy classes were given by Dr. Kalyas, who also continued to teach anatomy classes for Haydarpasa Military Medical Hospital Exercise and Operation School. In accordance with new design of the medical curriculum in 1893, we observe that anatomy classes were delivered by Major General Rasim and Major Ismail. In their classes they both used Jamin's 2 Volume non-illustrated Turkish translations and Sappeny's illustrated books, which were translated by Major General Mazhar. Besides mentioned above some of military medical doctors such as Mr.Yusuf Rami, Mr. Hikmet, $\mathrm{Mr}$ Rifat Husamettin, Mr. Nurettin Ali, Mr Kose Tevfik and Mr.
Ismail Hakki made great contributions to peculiarly anatomy education and broadly medical science in country and they deserve to be commemorated as scientists who put their stamp in development of Turkish Anatomy Education.

Keywords: History of medicine, Military medicine, Turkish medicine history, Tibbiye-i Sahane

\section{0-12}

\section{An ancient anatomic variation: bilateral elongated styloid process of cranium}

Özdemir MB* , Okunak $M^{* *}$, Köseler $A^{* * *}$, Şimşek $C^{* *}$, Atalay $\mathrm{E}^{* * *}$, Yonguç GN*

*Department of Anatomy, Faculty of Medicine; Pamukkale University, Denizli, Turkey; ${ }^{* \star}$ Faculty of Science and Letters, Part of Archaelogy; Pamukkale University, Denizli, Turkey; ***Department of Biophysics, Faculty of Medicine, Denizli, Turkey

The elongated stylohyoid process has been still presented considerable anatomic variability in nowadays. We report here an ancient cranium with bilateral elongated styloid process; $3,3 \mathrm{~cm}$ at the right side and $5,1 \mathrm{~cm}$ at the left side, found during the examination of excavated bones that belongs to 2000 years ago (first half of 1 . century AD) in old Roman city-Leodikya in Turkey. We determined the gender of the case, from the examination of the skeleton (especially skull and pelvic bones), as female. No other variation was observed. The reason for this is potentially partial ossifi-cation of the stylohyoid ligament, when considering the embryological development. Although elongation of the styloid process is common, it is important to report this ancient variation, in order to help to compare the bone variations between ancient human and modern human in terms of the contribution of genetic and environmental determinants.

Keywords: Elongated styloid process, anatomic variation, ancient

\section{0-13}

\section{Teeth variation of ancient Roman period population, Laodikeia}

Simșek N*, Çırak A**, Karaöz Arıhan S***

*Department of Anthropology, Faculty of Literature, Van Yüzüncü Yıl University, Van, Turkey; **Department of Classical Archaeology, Faculty of Literature, Karabük University, Karabük, Turkey; $* * *$ Department of Paleoanthropology, Faculty of Languages, History and Geography, Ankara University, Ankara, Turkey

Variations of external morphological properties of teeth in the context of anthropological studies have a great importance in determining proximity among different populations. Aim of this study is to assess Laodikeia population which is an ancient Anatolian population according to variations in teeth. Laodikeia ancient city is within the borders of Denizli province and excavations in this city have been performed since 2004. Unearthed Laodikeia skeletons dated to Roman period from the materials of this study. 1223 permanent teeth belonging to 115 adult, 62 


\section{S120 14th National Congress of Anatomy, 28 June-1 July 2012, Ankara, Turkey}

female and 53 male, were assessed for their teeth variations. Variations assessed in this study includes; supernumerary teeth, congenital absence of teeth, rotation of teeth, crowding of teeth, molar cusp pattern, extra cusp, shovel-shaped teeth, peg-shaped teeth, buccal pit, Taorodontism, enamel extensions and pearls, extra or missing roots, artificial deformation and first premolar with two roots in maxilla. In the Laodikeia population following the investigation of 115 individuals: shovel shaped teeth in 23 over 109 incisor of upper teeth $21.10 \%$, first premolar with two roots in upper jaw in 21 over 66 upper first premolar $31.82 \%$, buccal pit in 18 over 469 molar teeth $3.84 \%$ and rotation of teeth in 45 over 1223 teeth $3.68 \%$ were observed. In teeth from the skeletons of Laodikeia population, shovel-shaped teeth, first premolar with two roots in maxilla, buccal pit, and rotation of teeth variations were detected. In addition, teeth plan of big molars were also determined. There is not enough possibility for comparison of our data with other populations due to insufficient anthropological studies performed on ancient Anatolian populations. Future studies will increase the data on the subject and will provide more possibility for determining the proximity of Anatolian populations according to this perspective.

Keywords: Non-metric teeth variation, biological distance, dental morphology, Laodikeia

\section{0-14}

\section{Plastination from a medical student's perspective}

$\underline{\text { Bedük S}}^{*}$, Tunalı $\mathrm{S}^{* *}$

*Class of 2016, Faculty of Medicine, Hacettepe University, Ankara, Turkey; **Department of Anatomy, Faculty of Medicine, Hacettepe University, Ankara, Turkey

Approximately $70 \%$ of our body consists of fluids. Plastination depends on the replacement of the body fluids with reactive plastics by using special techniques. In this study, our objectives were to investigate the effects of formalin-fixation on plastinated specimens, as well as to assess the value of plastination in medical education and in the improvement of skills of medical students. One heart was directly proceeded into plastination, whereas one other underwent formalin-fixation first. Dehydration was performed in $-20^{\circ} \mathrm{C}$ cold acetone in an explosion-proof freezer. Specimens were then submerged into a S10/S3 solution for vacuum impregnation. Then, specimens were dissected to explore the atria and ventricles. Heart chambers and great vessels were trimmed and positioned. Plastination was concluded with final curing using S6 catalyst. Formalin-fixed specimen kept its original shape, had a light color and was completely odorless. Plastinated specimens have a great value in medical education. Such models enable anatomy practicing without exposure to formalin, even out of the dissection room and with one's bare hands. On the other hand, since we have extensive shortage of cadavers in our region; implementation of plastination into routine may insure a more effective use of cadavers, because plastinated specimens are durable. Furthermore, many medical students consider anatomy as a hard course; involvement of students in similar studies may contribute to a better anatomy understanding with having fun at the same time. In conclusion, the dissection I made and the laboratory experience I've got in this study will constitute a start up for my future studies. I opine that I had an immense experience in this regard.

Keywords: Plastination, medical education, formalin, fixation

\section{0-15}

\section{Tears on a crocodile's eyes}

\section{Türkoğlu MB*, Tunalı $\mathrm{S}^{* *}$}

*Class of 2016, Faculty of Medicine, Hacettepe University, Ankara, Turkey; **Department of Anatomy, Faculty of Medicine, Hacettepe University, Ankara, Turkey

The term "crocodile tears" arises out of an ancient myth that crocodiles use tears to lure their prey and then continue to shed tears as they devour them. This term is now used for the condition of copious lacrimation associated with eating. In this study our objective was to test whether this legend is correct and discuss paradoxical gustolacrimal tearing in detail. Literature reviewed for "crocodile tears", "paradoxical gustolacrimal tearing", "congenital aberrant tearing". Related manuscripts were reviewed systematically. Crocodile tears syndrome is a lacrimal hypersecretion disorder characterized by excessive tearing with gustatory stimulation while eating, drinking, or smelling food. It was first described in 1913 and since then many cases have been reported in the medical literature. This rare phenomenon usually follows a Bell's palsy, a traumatic facial paralysis or vestibular schwannoma surgery. However, it may occur congenitally with Duane's syndrome. It is often referred to as paradoxical gustolacrimal tearing or crocodile tears, so named by Bogorad, who said, "crocodiles cried when eating their prey." However, this term also refers to feigned or misleading hypocritical tearing. Although crocodiles have lacrimal glands, the secretion seems more directed to glands of eating than to ocular structures, so the implication may be inappropriate. Darwin believed that very few animals shed tears, with the possible exception of the India elephant and, questionably, some monkeys. There is a large spectrum of conditions that aberrant tearing accompanies, from congenital syndromes to traumatic facial paralysis or intracranial surgery complications. Each of them requires special attention for the diagnosis, pathophysiology and management.

Keywords: Crocodile tears, paradoxical gustolacrimal tearing, Bell's palsy, traumatic facial paralysis, vestibular schwannoma surgery

\section{0-16}

\section{Psychiatric medications and brain anatomy}

\section{Yücel K*, MacQueen GM**}

*Department of Anatomy, Faculty of Medicine, Yeditepe University, istanbul, Turkey; **Department of Psychiatry, Faculty of Medicine, Calgary University, Calgary, Alberta, Canada

We present data from the structural magnetic resonance imaging studies in which the effects of treatment with lithium $(\mathrm{Li})$, antipsychotics and antidepressants on the volumes of brain struc- 
tures have been investigated. We also provide our findings regarding the effects of $\mathrm{Li}$ and antidepressants particularly on hippocampus and anterior cingulate cortex (ACC) volumes. Through PubMed, we made several searches by using the following keywords:"hippocampus and lithium", "lithium, MRI, volume and bipolar", "lithium, volume, and bipolar", "antidepressant, volume and MRI", "antipsychotic, volume, and MRI". We and others have reported that reatment with $\mathrm{Li}$ is associated with increased hippocampal volume in bipolar patients. Additionally, increases in the amygdala, thalamus, superior temporal gyrus, and ACC are apparent following lithium treatment. Brain structures related to the effects of antipsychotic treatment show a wider spectrum. These structures include whole brain, ventricules, pituitary gland, insula, hippocampus, superior temporal gyrus, cingulate gyrus, frontal lobe, and basal ganglia structures. We found increased subgenual prefrontal cortex volume in remitted patients with major depression and larger posterior hippocampal pretreatment volumes in depressed for patients who achieved remission after eight weeks of antidepressant treatment. A positive treatment response to antidepressants is correlated with changes in the volumes of orbitofrontal cortex, inferior temporal gyrus, ACC, temporo-parietal cortex,hippocampus, and insula. Longitudinal studies with careful clinical follow up are required to confirm the relations between treatment with psychotropic medication and changes in specific brain structures.

Keywords: Psychotropic drugs, structural MRI, volume

\section{0-17}

\section{Evaluation of morphometric development of intracranial part of central nervous system during fetal period via magnetic resonance imaging}

$\mathrm{Uğuz} \mathrm{C}^{*}$, Malas MA**, Şener $\mathrm{EH}^{* * *}$, Sulak $\mathrm{O}^{* * * *}$

*Department of Anatomy, Faculty of Medicine, Muğla University, Muğla, Turkey; **Department of Anatomy, Faculty of Medicine, Izmir Katip Çelebi University, Izmir, Turkey; $* * \star$ Department of Nursing, School of Health, Mehmet Akif Ersoy University, Burdur, Turkey; $* * * *$ Department of Anatomy, Faculty of Medicine, Yıldırım Beyazıt University Ankara, Turkey

We aimed to gather morphometric data through MRI images in order to evaluate development of central nervous system throughout fetal period. One hundred and twenty fetuses (63 male and 57 female), aging 12 to 40 weeks, with no external pathology or anomaly chosen for the study. Fetuses were grouped based on gestational weeks, months and trimesters. First, general external parameters of fetuses were measured. Then, images obtained from sagittal, axial and coronal axes using Magnetic Resonance Imaging (MRI). Cranium, cerebrum, cerebellum, ventricles and truncus cerebri measurements from 2D images and also volume measurements of hemispherum cerebri were evaluated. Means and standard deviations calculated from the parameters which grouped by gestational weeks, months and trimesters. No significant difference found between sexes or between right or left sides ( $p>0.05$ ) among all parameters. There was significant difference between gestational ages $(\mathrm{p}<0.001)$. Our findings will be helpful to evaluate the development of cen- tral nervous system, to detection of local anomalies and to choose treatment options in departments of obstetrics and gynecology, pediatrics and fetopathology.

Keywords: Central nervous system, human fetus, intracranial nerve system, fetal development, anatomy

\section{0-18}

Is there relation between Cormack-Lehane
classification and neck anthropometry?

Y1lmaz ME*, Çolak A*, Hekimoğlu S*, Y1lmaz A**

*Department of Anestesiology and Reanimation, Faculty of Medicine, Trakya University, Edirne, Turkey; **Department of Anatomy, Faculty of Medicine, Trakya University, Edirne, Turkey

Cormack-Lehane (CL) classification is commonly used to predict the difficult airway. During direct laryngoscopy, the laryngeal view was graded using CL classification. CL grades III and IV were considered difficult visualization. The purpose of study is to investigate the anthropometric measurements of head and neck in relation to the Cormack-Lehane classification. 603 adult patients (238 male, 365 female) submitted for elective surgery under general anesthesia were included in this study. Age, height, weight, thyromental distance, sternomental distance, neck circumference, neck depth and bigonial distance were recorded during preoperative evaluation. CL was used for visualization of the larynx. CL classification was made by anesthetist and the anthropometric measurements were made by anatomist. The number and duration of attempts at each tracheal intubation were recorded. There was not any significant correlation between CL and age, height, weight, neck depth and bigonial distance $(p>0.05)$. But thyromental distance, sternomental distance, neck circumference measurements showed statistical significant effect on the CL $(\mathrm{p}=0,000)$. Cormack and Lehane classification is the most valuable test for predicting difficult intubation. CL is not used as preoperative bedside tests to predict a difficult airway. Therefore we need new easy applicable methods. According to our results, thyromental distance, sternomental distance and neck circumference usable instead of CL.

Key words: Cormack-Lehane classification, neck anthropometry

\section{0-19}

\section{Evaluation of the foot antropometric measurements for sex determination and stature estimation}

Zeybek G*, Ergür İ, Demiroğlu Uyanıker Z**, Kosay $\mathrm{C}^{* * *}$, Dalgıç $M^{* * * *}$, Şen $F^{* * * *}$

*Department of Anatomy, Faculty of Medicine, Dokuz Eylül University, izmir, Turkey; **Department of Forensic Medicine, Faculty of Medicine, Dokuz Eylül University, Izmir, Turkey; ***Department of Orthopedy and Traumatology, Faculty of Medicine, Dokuz Eylül University, Izmir, Turkey; $* * * \star$ Forensic Medicine Institute, İmir, Turkey

The anatomic consistency between the bone, joint and muscle of the foot constitutes the individual foot structure and shape. The 


\section{S122 14th National Congress of Anatomy, 28 June-1 July 2012, Ankara, Turkey}

morphologic, morphometric and antropometric features of the structure and shape of the foot are important to develop alternative methods for identification studies in forensic science. This study aims at developing models for sex determination and stature estimation through foot measurements when necessary. We collected the data from 89 forensic autopsies (31 women, 58 men) at Council of Forensic Medicine İzmir Group Chairmanship and measured stature and the right and left feet. The descriptive statistics of all the measurements have been presented and the comparison of measurement values between genders has been evaluated with t-test analysis. The relation between foot measurements and stature has been determined via pearson correlation analysis. By using logistic regression analysis and multiple regression analysis for sex determination and stature estimation respectively, models were formed. The lengths of navicular and medial cuneiform bones, and medial midfoot lengths of right and left feet did not show significant difference between the genders and did not have significant correlation with stature. Between the genders, right and left feet measurement means showed significant differences, which were used to form a model for sex determination. Stature estimation models were presented for all groups with significant correlation between stature and foot measurements. Sex determination models had correct estimation rates of $92,1 \%$ and $85,4 \%$ by using right and left feet measurements respectively. Stature estimation model had minimum $14,89 \mathrm{~cm}$ deviation in women and $7,50 \mathrm{~cm}$ deviation in men. The models without gender difference could calculate the stature with $8,5-10 \mathrm{~cm}$ deviation.

Key words: Foot, identification, stature estimation, sex determination, regression analysis

\section{$0-20$}

"Ear plug technique" to reduce the gag reflex during dental treatment procedures

Cakmak YÖ*, Özdoğmuş Ö ${ }^{* *}$, Günay $\mathrm{Y}^{* * *}$, Gürbüzer B****, Tezulas $\mathrm{E}^{* * * * *}$, Karaalioğlu $\mathrm{O}^{* * *}$, Kaspar $\mathrm{C}^{* * * * * *}$, Hacıŏlu $\mathrm{H}^{\star * * * * * * *}$

*Department of Anatomy\&Acupuncture Program for Physicians, Faculty of Medicine, Yeditepe University, Istanbul, Turkey; ${ }^{* *}$ Department of Anatomy, Faculty of Medicine, Marmara University, istanbul, Turkey; $* * *$ Department of Dentistry, Section of Prosthodontics, Gülhane Military Medical Academy, Haydarpaşa Training Hospital, istanbul, Turkey; ****Department of Dentistry, Section of Oral and Maxillofacial Surgery, Gülhane Military Medical Academy, Haydarpaşa Training Hospital, Istanbul, Turkey; *****Dental Academy, Private Practice, Istanbul, Turkey; $* * * * * *$ Department of Biostatistics, Faculty of Medicine, Yeditepe University, Istanbul, Turkey; $* * * * * * *$ Institute of the Neurological Sciences and Department of Anatomy, Faculty of Medicine, Marmara University, istanbul, Turkey

To evaluate the efficiency of a simple ear plug as an external ear canal skin (which is a referred pain zone of oropharyngeal cancers) stimulator to suppress the profound gag reflex(GR) during dental treatments and as a second step of the study to map the suppressed oropharyngeal areas with the aid of ear plug application. Our prospective randomized controlled study consists of two steps. At the first step of the study, 90 patients who have GR during the upper alginate impression procedure were randomly allocated to a study group, a sham group and a control group for evaluating the efficiency of the earplug technique. At the second step of the study, at which 20 new patients with GR included, to map the GR suppression areas (if the results of the step one found to be statistically significant). The results of the first step of the study demonstrated that the GR severity can be reduced in the ear plug group but not in the sham and the control group. The results of the second step of the study (mapping) demonstrated that ear plug application is an effective technique for reducing the GR derived from the upper palate, upper uvula and from the tongue but not from the posterior wall of oropharynx. Our results revealed that ear plug technique is a powerful technique to reduce the GR that is triggered from the trigeminal nerve distribution zones.

Key words: Gag reflex, ear, trigeminal, glossopharyngeus

\section{0-21}

Comparison of micro-computed tomography and histomorphometry for evaluation of bone architecture

\author{
Firat A*, Tatar $\dot{I}^{*}$, Kaymaz FF ${ }^{* *}$, Erbil KM* Çelik $\mathrm{HH}^{*}$ \\ *Department of Anatomy, Faculty of Medicine, Hacettepe University, \\ Ankara, Turkey; ${ }^{* *}$ Department of Histology and Embriyology, Faculty \\ of Medicine, Hacettepe University, Ankara, Turkey
}

Micro-computed tomography (micro-CT) is a non-invasive technique for the analysis and evaluation of the three-dimensional bone architecture. Aim of this study is to compare the results of the micro-CT and routine histologic evaluations of bone tissue, decalcified bone tissue and paraffin-embedded bone tissue. In this study, we dissected the tibia and fibula of a rat bilaterally. We obtained micro-CT images of these bones in transverse and longitudinal sections and then we performed routine histologic procedures for the same sections. In the second step we decalcified the bones and then performed the micro-CT and histologic procedures. In the last step we performed microCT to the paraffin embedded decalcified bone tissues and compared the images with the histologic preperations. Micro-CT images and histologic preparations of the fresh bone, decalcified bone and paraffin embedded bone are analysed and compared with each other for the comparison of these techniques in different tissue procedures. Micro-CT is an important technique for imaging and quantifying the bone tissue non-destructively in three dimensions. In order to help the researchers for their studies with micro-CT, we compared the results of the images with micro-CT with the gold standart histomorphometry.

\section{Keyword: Micro-CT}

\section{$0-22$}

\section{Effects of prenatal stress and maternal separation during lactation on depressive-like behaviors of Sprague-Dawley rats}

Söztutar E, Ulupinar E

Department of Anatomy Faculty of Medicine, Eskişehir Osmangazi University, Eskişehir, Turkey 
Exposure to early life adverse events may increase vulnerability to psychopathology in adult life. This study was conducted to examine depression-like behaviors in animals exposed to stress throughout prenatal and lactation period. Rats in the experimental group were exposed to stress by keeping dams immobile for 3 hours/day at prenatal period (14-21. embryonic days) and maternal separation for 3 hours/day after birth (221. postnatal days). According to anxiety levels, pups were divided into low or high anxiety groups $(n=8$ each group and gender) by application of the elevated plus maze test at postnatal day 30 and 60. Any application was made to the control group. Levels of spontaneous locomotor activity and susceptibility to depression were evaluated by activity meter and the Porsolt's swimming test (PST), respectively. Duration of struggling and immobility were recorded by a stopwatch, and the number of diving was counted in the first 5-minute periods of PST, on the second day. Differences between groups were compared by one-way analysis of variance. Although activity levels of low-and high-anxious female offspring were significantly lower in the stress group compared to controls, their susceptibility to depression was not significantly different. In males, on the other hand, despite no changes in the activity levels, immobility duration in the PST was significantly $(\mathrm{p}<0.01)$ longer in the low-anxious animals than other groups. Exposure to early life stress leads to adverse effects on depression-like behaviors in a manner dependent on gender, affecting male rats more significantly.

Keywords: Porsolt's swimming test, prenatal stress, maternal separation, depression, activity meter

\section{0-23}

Identification of the cortical, subcortical and mesencephalic afferent projections of the thalamic mediodorsal mucleus in rats

Cavdar S* ${ }^{*}$ Hacioğlu $\mathrm{H}^{* *}$

*Department of Anatomy, Faculty of Medicine, Koç University istanbul; **Department of Anatomy, Faculty of Medicine, Marmara University, istanbul**

Mediodorsal nucleus has a functional importance for several diseases. The aim of this study is to identify the connections of mediodorsal nucleus and to light its importance in the neuronal network for these diseases. Flouroscent retrograd Floro-Gold is stereotaxically injected to the mediodorsal nucleus of Wistar albino rats. Animals are decapitated with perfusion fixation method after 7-10 days. 40İm thick serial sections are taken from brains and connections identify via florescent microscope. Thalamic mediodorsal nucleus connections; 1 . Cortical; motor (especially head region), cingulate, somatosensory and prelimbic cortex. These cortical areas have connections especially from layer V. 2. Subcortical; Caudate nucleus and putamen, globus pallidus, diagonal band and thalamus paraventricular nucleus. 3. Mesencephalic; Substantia nigra, mecensephalic reticular nucleus. All stimuli that lead to cortex are mediated by thalamus. Mediodorsal nucleus is a higher-order thalamic nucleus. There is a cortico-thalamo-cortical pathway for MD, however the exact functions of that pathway are not known, it is thought that it may play a role in complex gating mechanisms. The mediodorsal nucleus with its position plays a role in the network cycles of the epilepsy, schizophrenia and Parkinson's diseases. It may play a role in initiation, modulation and synchronization of epileptic seizures. In schizophrenia, damage in connections of mediodorsal-prefrontal cortex leads an important pathology and for Parkinson's disease there is an important neuronal loss in mediodorsal thalamus. The knowledge of mediodorsal nucleus connections will considerably help to find out the mechanisms of the diseases.

Key words: Mediodorsal nucleus, thalamus, Floro-Gold, afferent

\section{0-24}

\section{Expression of NADPH-d in the vagal nuclei of the chronic esophagitis model in rats}

Şehirli US*, Tuğtepe $\mathrm{H}^{* *}$, Kirazlı O* , Özkan $\mathrm{M}^{*}$, Dağlı TE** *Department of Anatomy, Faculty of Medicine, Marmara University, istanbul, Turkey; **Department of Pediatric Surgery, Faculty of Medicine, Marmara University, Istanbul, Turkey

The reflux of acidic gastric contents and prolonged exposure of esophageal mucosa to gastric contents leads to esophagitis. Although esophagitis is a common health problem the changes occurring during esophagitis in the central nervous system are not well defined. It was suggested that during esophageal acid exposure nitric oxide synthase containing neuronal cells are activated to modulate esophageal reflux. This study aims to understand the response of the vagal nuclei to the chronic esophagitis. In this study, there were case, control and treatment groups. The chronic gastroesophageal reflux rat model was created by a partial gastric outlet obstruction in case and treatment groups. 4 weeks after surgery the rats in case group were sacrificed. The rats in treatment group were treated with ranitidine to eliminate the effect of surgery on the nuclei for four weeks. Histopathologically, chronic reflux esophagitis was observed in all specimens of the reflux group. The esophageal changes in the treatment group were not significant. The control group showed no changes related to esophagitis. The serial sections of brainstem of all groups were cut and NADPH diaphorase staining which selectively stains the nitric oxide synthase containing neurons was done. The number of the NADPH diaphorase stained neurons in the nucleus vagalis dorsalis and nucleus tractus solitarius was counted. The staining in the nucleus vagalis dorsalis and nucleus tractus solitarius were evaluated. There were statistically significant differences between case and control, case and treatment groups in both nuclei $(\mathrm{p}<0.05)$. The difference between control and treatment groups was not statistically significant. The increased nitric oxide expression in the nucleus vagalis dorsalis and nucleus tractus solitarius was most probably due to changed esophageal motility.

Keywords: Esophagitis, NADPH-d, nucleus tractus solitarius, nucleus vagalis dorsalis, nitric oxide synthase 
S124 14th National Congress of Anatomy, 28 June-1 July 2012, Ankara, Turkey

\section{0-25}

Protective effect of insulin and glucose in different concentrations on the astrocyte death induced by penicillin on the primer astroglial cell line

Özdemir $\mathrm{MB}^{*}$, Akça $\mathrm{H}^{* *}$, Tokgün $\mathrm{O}^{* *}$, Demiray A*, Fenkci $S^{* * *}$, Becerir $\mathrm{C}^{\star * * *}$, Erdoğan $\mathrm{C}^{\star * * * *}$

${ }^{*}$ Department of Anatomy, Faculty of Medicine, Pamukkale University, Denizli, Turkey; ${ }^{* *}$ Department of Medical Biology, Faculty of Medicine, Pamukkale University, Denizli, Turkey; $* * *$ Department of Endocrinology, Faculty of Medicine, Pamukkale University, Denizli, Turkey; $* * * *$ Department of Pediatrics, Faculty of Medicine, Pamukkale University,

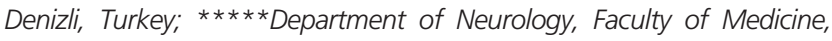
Pamukkale University, Denizli, Turkey

Astrocytes perform many functions in the brain and spinal cord. Glucose metabolism is important for astroglial cells and astrocytes are the only cells with insulin receptors in the brain. The common antibiotic penicillin is also a chemical agent that causes degenerative effect on neuronal cell. The aim of this study is to show astroglial cell survival effect of insulin and glucose in different concentrations on the astrocytes death induced by penicillin on primer astrocyt cell line. It is well known that intracranial penicilin treatment causes neuronal cell death and it is used for experimental epilepsy model commonly. Previous studies showed that insulin and glucose might protect neuronal cell in case of right concentrations. But, there is no data about the effect of penicilin on astrocyte and astroglial cell protection of insulin and glucose. For this purpose, 7-day-old newborn rats' brain were dissected, then mechanically dissociated to astroglial cell suspension and finally grown in culture medium as described previously. Culteres were maintained for 2 weeks prior to being used in these experiments. Different concentrations of insulin (0 $\mathrm{nM}, 1 \mathrm{nM}, 3 \mathrm{nM})$ and glucose $(0 \mathrm{mM}, 3 \mathrm{mM}, 30 \mathrm{mM})$ were used in mediums without penicillin and with $2500 \mathrm{M}$ penicillin. Penicillin decreased the viability of astroglial cell seriously. The highest cell viability was in medium with $3 \mathrm{nM}$ insulin- $3 \mathrm{mM}$ glucose concentration when medium was without penicilin. But, in medium with penicillin, the best cell survival was in medium with $1 \mathrm{nM}$ insuline-glucose free concentration. Insuline and glucose were protective against the penicillin on primer astrocyt cell line. Interestingly, cell survival was up to concentrations of insulin and glucose strongly. The results of this study will help to explain cerebrovasculer pathologies paralell to insulin and glucose conditions of patient after intracranial injuries.

Keywords: Astrocyte, penicilin, insulin, glucose

\section{0-26}

\section{Isolated bilateral congenital external auditory canal} atresia: case report

Tetiker $\mathrm{H}^{*}$, Sahan $\mathrm{M}^{* *}$, Deveer $\mathrm{M}^{* * *}$, Çullu $\mathrm{N}^{* * *}$

*Department of Anatomy, Faculty of Medicine, Muğla University, Muğla, Turkey; ${ }^{*}$ Department of Otolaryngology, Faculty of Medicine, Muğla University, Muğla, Turkey; **Department of Radiology, Faculty of Medicine, Muğla University, Muğla, Turkey

External auditory canal atresia occurs one of 10000-20000 births and is bilateral in approximately one fifth of patients. The external and middle ear develops from first and second branchial arcs, and first branchial cleft simultaneously. The external auditory canal recanalises at 4- 7 months. In embryo pathogenesis in this period may result in isolated external congenital auditory canal atresia. Case was evaluated with physical examination, audilogical examination and High-resolution computed tomography. A Toshiba Asteion Multislice CT used in the radiological examination. CT sections of $2 \mathrm{~mm}$ thickness were obtained in axial projections. Atresia was graduated classification of Schuknect, Altmann and Jahrsdoefer et al. In this study isolated bilateral congenital external auditory canal atresia of the case was evaluated clinical and radiological perspectives. Physical examination, bilateral external ear canal and meatus exist but short and cone form occluded inside. Temporomandibular joint, auricula and tragus of patient was fully developed and there was no facial hypoplasia. In audiological examination $40 \mathrm{~dB}$ (right ear) and $37 \mathrm{~dB}$ (left ear) conductive hearing loss was detected. In temporal bone CT examination, bilaterally middle ear structure, mastoid aeration and auditory ossicles were intact but at the level of tympanic membrane bilaterally external canals were obstructed with bone and soft tissue density. On the left medial- lateral thickness and antero- posterior diameter of the canal were $4,8 \mathrm{~mm}$ and 4,7 $\mathrm{mm}$ respectively. On the right medial- lateral thickness and antero- posterior diameter of the canal were 7,1 $\mathrm{mm}$ and 5,5 $\mathrm{mm}$ respectively. A result of final findings, atresia was graduated as tip A in Schuknect classification, type I in Altmann classification and got 9 points in Jahrsdoefer et al classification.

Keywords: Isolated bilateral congenital external auditory canal atresia, high-resolution CT 


\title{
Poster Presentations
}

\author{
(P-1 - P-77)
}

\section{P-1}

\section{Changes and development of national congress of anatomy over the course of time}

\author{
Songür A*, Turamanlar O** Gönül $\mathrm{Y}^{*}$, Akçer $\mathrm{S}^{*}$, Ayçiçek $\mathrm{H}^{*}$ \\ Department of Anatomy, Faculty of Medicine, Afyon Kocatepe \\ University, Afyonkarahisar, Turkey; **Sinanpaşa Family Health Center, \\ Afyonkarahisar, Turkey
}

In our study, the changes between topics of presentation, people who published them and their material-methods were examined over the years by using the books which were published in previous years at the National Congresses of Anatomy. In this study, tables were created considering the years and they were assessed quantitatively by examining the abstract books from the National Anatomy Congress between 2000-2010. In these tables, the numbers of presentations as oral or poster, published presentations, their universities or institutions, their faculties and departments were examined separately. Additionally, topics of papers, studied materials and differences between its system or limb in studies and presentation methods were investigated separately. According to obtained data, the distribution of oral presentation had increased quantitively; however, poster-type declarations had stayed same over the time. The number of participating universities and departments, macro- and micro-anatomical studies and radiological studies (X-Ray, CT and MR) had showed an increase. Anthropometric studies had concentrated in the trunk and lower extremities. Microanatomic studies on human had increased over the years compared to the experimental animals; additionally, it was seen that studies were concentrated on the nervous system. In this study, we believe that studies which were presented in National Anatomy Congress will be useful for academicians for their future studies.

Keywords: Anatomy, congress, national, presentation

\section{P-2}

Protective effect of alpha lipoic acid on rat sciatic nerve ischemia reperfusion damage

Turamanlar $\mathrm{O}^{*}$, Özen $\mathrm{OA}^{* *}$, Songür $\mathrm{A}^{*}$, Yağmurca $\mathrm{M}^{* * *}$, Akçer $\mathrm{S}^{*}$, Mollaoğlu $\mathrm{H}^{* * * *}$, Aktaş $\mathrm{C}^{* * * * *}$

*Department of Anatomy, Faculty of Medicine, Afyon Kocatepe University, Afyonkarahisar, Turkey; ${ }^{*}$ Department of Anatomy, Faculty of Medicine, Namık Kemal University Tekirdağ, Turkey; ${ }^{* * *}$ Department of Histology Embriyology, Faculty of Medicine, Fatih University, Ankara, Turkey; $* * * *$ Department of Physiology, Faculty of Medicine, Sifa Universitesi, Izmir, Turkey; $* * * * *$ Department of Histology and Embryology, Faculty of Medicine, Namık Kemal University, Tekirdağ, Turkey
In this study, protective effect of alpha-lipoic acid (ALA) on sciatic nevre following ischemia-reperfusion (I/R) in rats was investigated by using light microscopy (HE and immunohistochemical methods) and biochemical methods (SOD and GSHPx enzyme activities as well as MDA and NO levels). In this study, a total of fourty-two Sprague-Dawney male rats were divided into six groups. Ischemia was carried out the abdominal aort starting from the distal part of the renal vein for two hours followed by reperfusion for three hours at I/R groups. As a result of histopatological investigations, vascular congestion and proliferation as well as myelin degeneration were found to be increased significantly in the sciatic nerve tissues of ischemia group; however, these findings were found to have diminished significantly in various degrees depending on the ALA groups by giving ALA (100 mg/kg). Fibronectin, an important component of extracellular matrix in the developing periferic nervous system, was observed to have increased significantly in ischemia group; on the other hand, it was observed to have decreased in paralel to the doses in the ALA given groups. In biochemical analyses; SOD and GSHPx enzyme activities were found to be lower by ischemia, however, in the ALA group being given $100 \mathrm{mg} / \mathrm{kg}$ of ALA, SOD and GSHPx enzyme activities were found to have increased. In the ALA given groups, increased MDA levels by I/R was observed to have decreased significantly in a dosedependent manner. The decreased NO level by I/R, was seen increased in ALAgiven groups, though it was not statistically significant. The fact that fibronectin increase observed after ischemia/reperfusion of rat sciatic nerve is reduced after administration of ALA indicates that fibronectin's function to reconnect cut nerve segments and regenerate nerves is more prominent than its function in tissue healing after ischemia. ALA administered before ischemia decreases MDA and increases SOD and GSHPx. We think that these positive changes show that ALA may protect against the pathological changes in ischemic nerve and may be used to devise more efficient treatments.

Keywords: Alpha-lipoic acid, ischemia-reperfusion, protective effect, rat, sciatic nerve

\section{P-3}

\section{An anatomical look at the historical development of} cesarean operation

Turamanlar $\mathrm{O}^{*}$, Songür A**

*Family Health Center of Sinanpaşa, Afyonkarahisar, Turkey; **Department of Anatomy, Faculty of Medicine, Afyon Kocatepe University, Afyonkarahisar, Turkey 


\section{S126 14th National Congress of Anatomy, 28 June-1 July 2012, Ankara, Turkey}

Cesarean operation is a laparotomic procedure in which an incision is made through abdominal wall and uterus in order to deliver babies when vaginal delivery is impossible. It is performed frequently when the life of the baby and/or the mother is at risk. Cesarean delivery rate in our country is $37 \%$ which is more than recommended by World Health Organization (WHO) and it is also high compared to other countries. First Cesarean section which the mother survived was performed in 1500s. However, mother death rates in cesarean section was still as high as $85 \%$ even in the late 1800 s. Deaths usually occurred due to uncontrollable bleeding during the operation. But, as the anatomical knowledge improved, cesarean section technique was refined as well as other surgical procedures and modern standards were achieved with the aid of developments in anesthesiology. The idea expressed in the frequently quoted saying "Nulla Medicina Sine Anatomia" (There is no medicine without anatomy) is justified whenever the historical aspect of any topic in medicine is explored. Our aim is to explore historical development of cesarean section, which is a widely used surgical procedure, in the context of improvements in anatomy.

Keywords: Cesarean operation, history of medicine, anatomy

\section{P-4}

\section{Substernal goiter: a case report}

Başol $N^{*}$, Taş $\mathrm{U}^{* *}$, Ayan $\mathrm{M}^{*}$, Esen $\mathrm{M}^{*}$, Cor AY*, Kablan $\mathrm{A}^{*}$, Alatl $1 \mathrm{~T}^{*}$

*Department of Emergency Medicine, Faculty of Medicine, Gaziosmanpaşa University, Tokat, Turkey; *Department of Anatomy, Faculty of Medicine, Gaziosmanpaşa University, Tokat, Turkey;

In this study, we are presenting a case, which has come to the emergency department with shortness of breath and substernal goiter, purposing to draw attention to this rare cause of type 2 respiratory failure. Substernal goiter is a thyroid gland disorder that would occupy within the chest cavity and would origin from the mediastinal space, mostly occurs as cervical goiter growth in the upper mediastinum and can lead to symptoms of compressing. A sudden deterioration in adequate gas exchange ability in the respiratory system is defined respiratory failure. Type 1 respiratory failure is characterized by deterioration in primary oxygenation; however type 2 respiratory failure is characterized by a sudden increase in carbon dioxide pressure. The causes of hypoventilation in type 2 respiratory failure can be counted as brainstem respiratory center depression, upper motor neuron injury, anterior horn cell damage and respiratory muscle disease and chest wall deformities. 76-year-old female patient was admitted to our hospital with shortness of breath. On physical examination, her lips were cyanotic and especially in inspirium there was prominent stridor. The thyroid gland was palpable (bilateral +2 ). In the patient blood gas, hypercarbia was detected and the patient was diagnosed with type 2 respiratory failure.

Keywords: Thyroid gland, substernal goiter, respiratory failure

\section{P-5}

Evaluation of relationship between fracture and bleeding in the patients of head trauma with brain hemorrhage or fracture

Taş $\mathrm{U}^{*}$, Ayan $\mathrm{M}^{* *}$, Karaman $\mathrm{T}^{* * *}$, Başol $\mathrm{N}^{* *}$, Ortak $\mathrm{H}^{* * * *}$, Esen $M^{* *}$, Başol $G^{* * * *}$

*Department of Anatomy, Faculty of Medicine, Gaziosmanpaşa University, Tokat, Turkey; **Department of Emergency Medicine, Faculty of Medicine, Gaziosmanpaşa University, Tokat, Turkey; ***Tokat State Hospital, Clinic of Anesthesiology and Reanimation, Tokat; $* * * *$ Department of Ophthalmology, Faculty of Medicine, Gaziosmanpaşa University, Tokat, Turkey; ****Department of Educational Research and Evalution, Faculty of Medicine, Gaziosmanpaşa University, Tokat, Turkey

In this study it is aimed to determine the most common bleeding type and it's localization in the patients, the type of the trauma and as to whether there is a fracture in patients attended to emergency department of our hospital because of head trauma. This study performed by the evaluation of the patient information from Enlil-HIS data system of our hospital and the patient files of the patients attended to emergency department because of head trauma. The study involed 47 major head trauma patients, 15 female (68.1\%) and 32 male (31.9\%), with either fracture or bleeding or both of them in cranium. As a result, the most common cause of major trauma was traffic accidents and the second rate was falling down. It is determined that major trauma was higher in males than in females, and $14.9 \%$ of them resulted in death. Majority of the deaths was over 65 -age $(42.8 \%)$. Head traumas with fractures and accompanying bleeding was significantly high (38.3\%). There was no statistically significance difference in terms of hemorhage between with fracture or without $(p>0.05)$. Most common localization of the bleedings were frontal, frontoparietal and temporal regions and the majority of them were subdural (25.5 $\%)$ and subarachnoidal (23.4 \%) bleedings. It should be considered that possibility of brain hemorrhage in head trauma patients without fracture up to the possibility of fractured patients being admitted to emergency service.

Keywords: Head trauma, bleeding, fracture

\section{P-6}

\section{Panniculitis of the mesentery}

Başol $N^{*}, \underline{\text { Taş } U^{* *}}$, Ayan $M^{*}$, Esen $M^{*}$, Arslan $M^{* * *}$, Koç $\mathrm{I}^{*}$, Acu B****

*Department of Emergency Medicine, Faculty of Medicine, Gaziosmanpaşa University, Tokat, Turkey; **Department of Anatomy, Faculty of Medicine, Gaziosmanpaşa University, Tokat, Turkey; ***Clinic of

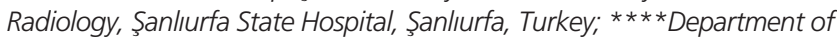
Radiology, Faculty of Medicine, Gaziosmanpaşa University, Tokat, Turkey

The mesentery is the double layer of peritoneum that suspends small intestine (jejunum and ileum) from the posterior wall of the abdomen. Superior mesenteric artery and its branches, accompanying veins, lymphatic, lymph nodes, the autonomic 
nervous plexuses, with a larger amount of connective tissue and varying amounts of fat tissue around the root of mesentery are located between to two layers of peritoneum. Mesenteric panniculitis is a benign disorder that characterized by fat necrosis, chronic inflammation and fibrosis. Although the etiology is not clear, it is associated with many diseases such as previous abdominal surgery, malignancies diseases. Mesenteric panniculitis, may be asymptomatic or accompanied by symptoms such as abdominal pain, abdominal distention, palpable mass and fever. Laboratory tests are non-specific in diagnosis. Although diagnosis of disease is usually with ultrasound and abdominal CT definitive, diagnosis is made by histopathological examination. Usually symptomatic treatments are performed in mesenteric panniculitis however immunosuppressive agents or steroids have also been reported to be effective. In this study, we report on three cases applied the emergency room with abdominal pain and diagnosed as mesenteric panniculitis, so we wanted to draw attention to the cause of this rare abdominal pain.

Keywords: Mesentery, panniculitis, abdominal pain

\section{P-7}

Sex comparisons with morphometric measurements in the proximal end of ulna

Başaloğlu $\mathrm{H}^{*}$, Tellioğlu $\mathrm{MA}^{*}$, Başaloğlu $\mathrm{HK}^{* *}$

*Department of Anatomy, Faculty of Medicine, Adnan Menderes University, Aydın, Turkey; ${ }^{*}$ Department of Histology and Embryology, Faculty of Medicine, Adnan Menderes University, Aydın, Turkey

In this study on proximal end of ulna with 5 sheets of morphometric measurements, aimed to the detection of gender-related differences. This study was examined by using total 60 ulna which belongs to 30 men and 30 women ulna and bones are taken from the bone laboratory of the Department of Anatomy, Adnan Menderes University, Aydin. The specimens consist of dry bone ulna from mature skeletons of 30 female (15 right, 15 left), 30 male (15 right, 15 left). Five measurements that were used by researchers previously were made on proximal end of ulna. Measurements for the millimeter caliper were used. Obtained data were analyzed with SPSS (14.0 version) program. Compliance with all of the normal distribution of data Kolmogorov-Smirnov test was applied. T-test was used to evaluate the differences between the sexes. Fisher's linear discriminant analysis with the help of metric values between the sexes was found that the best separation. The results of the present study demonstrate that In all parameters measured in the proximal end of ulna was significantly greater in males compared to that in females. When the measurements were analysed by t-test which showed a statistically significant difference between males and females $(p<0.05)$. Than all of the variables' Fisher linear discriminant function test 'applied to $80 \%$ of men and women $76.7 \%$ ' s correctly classified $78,3 \%$ of the whole group. It is concluded that mesurements from the proximal end of ulna can be used as a reliable sex marker. According to our results, all measurements from proximal end of ulna can be determined by sex \% 78.3. Metric data obtained in our study shall be useful in case of that sex of skeleton could not be determined morphologically.

Keywords: Ulna, morphometry, sex determination

\section{P-8}

\section{Effects of modafinil on pentylenetetrazol- induced} convulsive epilepsy in rats

\section{Özsoy $S^{*}$, Çakıl D*, Ekici F**}

*Department of Physiology, Faculty of Medicine, Gaziosmanpaşa University Tokat, Turkey; ${ }^{* *}$ Department of Physiology, Faculty of Medicine, Yıldırım Beyazit University Ankara, Turkey

Epilepsy is a chronic disorder caused by abnormal, sudden and excessive discharges of the neuron groups in the central nervous sistem. Modafinil, is an approved drug by Food and Drugs Administration (FDA) for narcolepsy and sleep-apnea syndrome. In this study, we aimed to investigate the dose dependent effects of modafinil on pentylenetetrazol model of experimental epilepsy; a widely used method for inducing convulsive epilepsy in rats. In this study, five experimental groups were administrated a single dose/day of modafinil for seven days (1, $2,4,45,180 \mathrm{mg} / \mathrm{kg}$, i.p), whereas the control group was administrated an equal amount of normal saline simultaneously, All groups were injected PTZ (80 mg/kg, i.p) 2 hours after the final dose and the epileptic seizure activity was evaluated. The seizure activity was determined by detecting the first jerk and major seizure onset period and the total major seizure period. When all groups were compared; it was detected that modafinil increases the onset period of the first jerk, decreases the total major seziure period at doses higher than $1 \mathrm{mg} / \mathrm{kg}$ and that there was no statistically significant difference between the major seizure onset periods in any groups. These results imply that modafinil can show a dose dependent antiepileptic effect on pentylenetetrazol-induced convulsive epilepsy in rats.

Keywords: Modafinil, pentylenetetrazole, epilepsy, generalized tonic-clonic seizures

\section{P-9}

\section{Relationship between the anthropometric} measurements and birth weight in newborns

Mutluay SD*, Bakır E*, Bozkır MG**

*Department of Obstetrics, Adana Health High School, Çukurova University, Adana, Turkey; **Department of Anatomy, Faculty of Medicine, Çukurova University, Adana, Turkey

This study was taken to determine the relationship between anthropometric measurements and birth weight (NWt) in newborns. For this purpose, measures such as head (HC), chest (CC), abdomen (AbC), mid-arm (MAC), calf (CCf) and thigh (TC) circumference, newborns height (NH), upper arm (UAl) and foot length $(\mathrm{Fl})$ were taking from neonates of the association between birth weights. The study carried out between October 2011-March 2012 in Adana Cukurova Women Delivery and 
Children's Hospital. The usefulness of anthropometric parameters as predictors of birth weight was studied in 212 (110 female, 102 male) newborns. The anthropometric parameters were measured within $24 \mathrm{~h}$ of delivery, using a fiberglass non-extendable measuring tape, with a width of $1 \mathrm{~cm}$. Hospital births showed a strong correlations (P0.005), $(\mathrm{r}=0.125)$, and also no significant difference between gender and weight (110 girl, 102 male) were observed ( $>>0.005)$. Anthropometric parameters are simple, practicable, quick, and reliable indicators for identifying birth weight and for predicting low birth weight.

Keywords: Anthropometry, newborn, birth weight

\section{P-10}

\section{The unilateral variation of musculus palmaris longus: case report}

Kurtoğlu E, Ertekin T, Nisari M, Karaca İ, Acer N

Department of Anatomy, Faculty of Medicine, Erciyes University, Kayseri, Turkey

Palmaris longus muscle (PLM) is a muscle of forearm. It takes origin from the medial epicondyle of humerus and lies medial to flexor carpi radial muscle. PLM is one of the muscles in upper limb that shows many variations. These variations show differences depending on race and gender as bilateral or unilateral. During routine cadaver dissection at Erciyes University Medical Faculty Department of Anatomy's laboratory a unilateral PLM tendon variation was found in a female cadaver aged 57 and weighted $98 \mathrm{~kg}$. While PLM found in cadaver's right forearm lay normally, it was observed that PLM found in the left forearm divided in two parts (tendons) nearly $5 \mathrm{~cm}$. away from the flexor retinaculum. We observed that lateral one of these tendons lay normally and inserted on the palmar aponeurosis, whereas the medial one inserted on the flexor retinaculum. We think that the knowledge about these variations of PLM may be useful in orthopedic surgery and also provides a very useful graft in tendon surgery.

Keywords: Palmaris longus muscle, variation, insertion.

\section{P-11}

\section{Visfatine expression in smooth and striated muscle tissues}

Arican RY*, Akkoyunlu G ${ }^{* *}$, Demir $N^{* *}$, Suzen LB ${ }^{* * *}$

*Department of Anatomy, Faculty of Medicine, Yakındoğu University Nicosia, Turkish Republic of North Cyprus; **Department of Histology and Embryology, Faculty of Medicine, Akdeniz University, Antalya, Turkey; ***Department of Anatomy, Faculty of Medicine, Akdeniz University, Antalya, Turkey

Visfatine is a novel adipokine reported by Fukuhara et all. in 2004. Visfatine has $52 \mathrm{KDa}$ weight and genomic chain with 491 aminoacids. The visfatine studies were usually performed in systemic blood. The aim of this study was to identify the localization of visfatine in smooth and striated musles immunohistochemically. Visfatine, causes inflammation in vascular smooth muscles related with the vascular dysfunction and some metabolic syn- dromes. The results of this study indicated that visfatin localizes in smooth tunica media muscles of ovarial vessels. The increase of the visfatin serum visfatin levels after exercise was reported in the literature. After the results of this tissue focused study the localization of visfatine in both smooth and striated muscle tissues are new comings for the literature. We observed pozitive visfatin immunreaction in both soleus muscle and smooth muscle cells of the tunica media layer of the ovarial vessels. In both tissues there were no visfatin immunreaction in negative control slides.

Keywords: Visfatine, smooth muscle, striated muscle, immunohistochemistry

\section{P-12}

\section{Relationship between coronary artery disease and coronary artery anomalies}

$\underline{\text { Aydar } \mathrm{Y}^{*}}$, Yazıc1 HU**, Birdane A**, Nadir A**

*Department of Anatomy, Faculty of Medicine, Eskişehir Osmangazi University, Eskişehir, Turkey; ${ }^{*}$ Department of Cardiology, Faculty of Medicine, Eskişehir Osmangazi University, Eskişehir, Turkey

Coronary artery disease (CAD) and its complications are the major cause of death among people worldwide. However, the number of the studies examining the potential relationship between the CAD and coronary artery anomalies (CAAs) is limited. In the present study we therefore aimed to investigate the frequency and types of CAAs and their correlation with CAD. We assessed retrospectively the coronary angiography records of 8100 patients admitted to the Department of Cardiology at Medical School of Eskisehir Osmangazi University. We defined and classified the CAAs according to the classification system described by Angelini et al. Moreover, we further compared the frequencies and features of the CAAs between CAD (-) and CAD (+) patients. We detected coronary artery anomalies and significant coronary artery disease in $3.33 \%$ and $64.4 \%$ of the study population, respectively. The percentage of anomalous coronary artery origin was significantly higher in CAD (-) patients than CAD (+) patients. The incidence of the absence of left main coronary artery (LMCA) and the left anterior descending artery originating from the right sinus of Valsalva or the right coronary artery was significantly higher in CAD (-) patients than CAD (+) patients. The present results indicated that CAD might be associated with CAAs. In particular, the absence of LMCA might reduce the development of CAD via regulating a more laminar blood flow in the coronary arteries.

Keywords: Coronary artery anomaly, coronary artery disease

\section{P-13}

Perineal ectopic testis: an unusual cause of perineal pain and empty scrotum

Akkoç $A^{*}$, Diramalı $M^{* *}$, Arifoğlu $\mathrm{Y}^{* * *}$

*Department of Urologia, Diyarbakır Teaching and Research Hospital, Diyarbakır, Turkey; **Department of Anatomy, Faculty of Medicine, Abant izzet Baysal University, Bolu, Turkey; ${ }^{* *}$ Department of Anatomy, Faculty of Medicine, Bezmialem Vakıf University, Istanbul, Turkey 
The testes are the primary reproductive organs or gonads in the male. In the early fetal period the testes are located posteriorly in the abdominal cavity. Their descent to the scrotum appears to be under hormonal control Testicular maldescence is the most common anomaly of the genitalia with incidence of $1.5 \%$. It is reported that $5 \%$ of maldescended testes are ectopic. Ectopic testis can be found at opposite side of the scrotum, suprapubic region, femoral canal, perineum, and anterior abdominal wall. The ectopic locations of the testes are associated with a number of complications like trauma, torsion and infertility. In this study a case study about perineal ectopic testis is presented. A 21 year old man was admitted to the urology clinic because of perineal pain while he is walking and sitting and right empty scrotum with an oval shaped soft mass found in the perineal region. By ultrasonography, a right perineal ectopic testis that is $3.8 \times 2.0 \times 2.2 \mathrm{~cm}$ in diameter and normally echogenity was demonstrated. Testis was fixed in the ipsilateral scrotum using the standard dartos pouch technique in the patient whom transrectal and urinary system ultrasound showed no pathology. Consequently, PET is certainly kept in mind in differential diagnosis of perineal pain and empty scrotum and also all of the cases should be researched in terms of PET.

Keywords: Orchiopexy, perineal, testicular ectopia

\section{P-14}

\section{The protective effects of omega-3 fatty acids on rat testicular tissue}

Zararsız $\dot{I}^{*}$, Kuş $\dot{I}^{* *}$, Davarcı $M^{* * *}$, Kuş $M^{* * * *}$, Kaman $\mathrm{D}^{* * * * *}$, Sarsilmaz $\mathrm{M}^{* * * * * *}$

*Department of Anatomy, Faculty of Medicine, Mevlana University, Selçuklu, Konya, Turkey; **Department of Anatomy, Faculty of Medicine, Balıkesir University, Balıkesir, Turkey; ***Department of Urology, Tayfur Ata Sokmen Medicine Faculty, Mustafa Kemal University, Antakya, Turkey; $* * * \star$ School of Health Sciences, Mehmet Akif Ersoy University, Burdur, Turkey; $* * * * *$ Department of Medical Biochemistry, Faculty of Medicine, Firat University, Elazı̆g, Turkey; ******Department of Anatomy, Faculty of Medicine, Şifa University, Izmir, Turkey

In this study, the protective effect of omega-3 fatty acids on testicular tissue was aimed to investigate at biochemical levels. Totally, 16 adult male Wistar rats were divided into two groups. Rats in Group I were used as control and only saline was given by intragastric gavage. Rats in Group II, 400 mg/kg dose $\omega-3$ fatty acids were given daily by intragastric gavage. At the end of the six-week experimental period, all rats were killed by decapitation. The testicular tissue specimens taken from animals, superoxide dismutase, glutathione peroxidase, malondialdehyde, enzyme activities were measured spectrophotometrically. In addition, blood testosterone levels were examined. In our study, $\omega-3$ fatty acids in rats were given a statistically significant increase in the levels of superoxide dismutase, and glutathione peroxidase a statistically significant decrease in malondialdehyde levels were determined when compared to control group. In addition, $\omega-3$ fatty acids in rats given a statistically significant increase in blood testosterone levels were observed. We concluded that $\omega-3$ fatty acid had favorable effects in rat testis tissue by preventing oxidative damage and increasing the level of testosterone.

Keywords: $\Omega-3$ fatty acids, testicle, testosterone, rat

\section{P-15}

\section{Effects of ginkgo biloba on biochemical and histopathological changes in the lungs of rats exposed to transfluthrin by inhalation}

Çakıl D*, Özsoy $S^{*}$, Çaylı $S^{* *}$, Taş $U^{* * *}$, Söğüt $E^{* * * *}$, Karaca $Z \mathrm{I}^{* *}$

*Department of Physiology, Faculty of Medicine, Gaziosmanpaşa University, Tokat, Turkey; ${ }^{* * D e p a r t m e n t ~ o f ~ H i s t o l o g y, ~ F a c u l t y ~ o f ~}$ Medicine, Gaziosmanpaşa Universty, Tokat, Turkey; ***Department of Anatomy, Faculty of Medicine, Gaziosmanpaşa University, Tokat, Turkey; $* * * *$ Department of Biochemistry, Faculty of Medicine, Gaziosmanpaşa University, Tokat, Turkey

Pyrethroid insectisides are known to cause neurotoxicity and increase oxidative stress in animal studies. Ginkgo biloba is an antioxidant agent that controls supression of apoptozis. In this study; we aimed to investigate the effects of ginkgo biloba on oxidative damage and apoptozis that may occur in the lungs of the rats exposed to mosquito repellent liquids including transfluthrin. 1 control and 4 experimental groups formed using 35 Wistar albino male rats. The experimental groups were administrated transfluthrin (by inhalation, $8 \mathrm{~h}$ /day), ginkgo biloba (100 mg/kg, i.p) and transfluthrin + ginkgo biloba for 4 weeks; respectively. Control group was administrated serum physiological with same volume at the same time. At the end of 4 weeks; all animals were sacrified under deep anesthesy. Tissue samples were taken from the lungs of all rats and MDA, NO, SOD and GSH-Px levels were measured biochemically. Also apoptotic indexes were calculated by Tunel (Terminal deoksinukleotidil transferaz mediated d-UTP nick end labeling) method to determine the apoptotic changes that might occur in the tissues. As a result of the Tunel method which provides to identify DNA fractures insitu; the apoptotic indexes were determined as $3.5 \%, 0.5 \%, 0.1 \%$ and $0.4 \%$ in the tissue samples from the lungs of control, transfluthrin, ginkgo biloba and transfluthrin + ginkgo biloba administrated groups; respectively. The oxidative stres markers and apoptotic indexes of transfluthrin group were significantly increased compared to all other groups. Also the oxidative stres markers and apoptotic indexes of the transfluthrin + ginkgo biloba coadministrated group were significantly decreased compared to transfluthrin group. Ginkgo biloba extract significantly decreases the oxidative and apoptotic damage in the lung of the rats caused by transfluthrine exposure by inhalation.

Keywords: Transfluthrin, pyrethroid, ginkgo biloba, oxidative stress, apoptozis 
S130 14th National Congress of Anatomy, 28 June-1 July 2012, Ankara, Turkey

\section{P-16}

Omega-3 fatty acids effect on kidney antioxidant defense (immune) system

Gülcen B*, Karaca Ö*, Kuş MA**, Kaman D ${ }^{* * *}$, Öğetürk $M^{* * * *}$, Kuş I*

*Department of Anatomy, Faculty of Medicine, Balıkesir University, Balıkesir, Turkey; **Health Collage, Mehmet Akif Ersoy University, Burdur, Turkey; ${ }^{* *}$ Department of Biochemistry, Faculty of Medicine, Firat University, Elazığ, Turkey; $* * * *$ Department of Anatomy, Faculty of Medicine, Firat University, Elazı̆̆, Turkey

In this research, omega-3 ( $\omega-3)$ fatty acids' protective effect on kidney tissue is biochemically investigated. For this purpose, 16 adult Wistar-Albino rats are divided into two groups and investigated. Rats in Group I are used as control group and they are only intragastrically gavaged with normal saline. However, rats in Group II are intragastrically gavaged daily with a dose of 400 $\mathrm{mg} / \mathrm{kg}$ fatty acid. All rats are killed by using decapitation method at the end of six week expreriment period. Kidney tissues collected from experimental subjects are spectrophotometrically investigated for Superoksit dismutaz (SOD), glutatyon peroksidaz (GSH-Px) and malondialdehit (MDA) enzime activities. It has been observed that SOD and GSH-Px levels are statistically increased for rats treated with $\omega-3$ fatty acid. Along with this, there had been a statistical decrease in their MDA levels compared to the results coming from control group subjects. It is cocluded that $\omega-3$ fatty acids prevent the oxidative damage in rat kidney tissue. These fatty acids have posivite antioxidant effect, thus protect kidney tissue.

Keywords: Omega-3 fatty acid, kidney, rat

\section{P-17}

The protective effects of melatonin hormone against experimental cadmium toxicity in the liver: an immunohistochemical study

Gülcen B*, Karaca Ö*, Kuş MA**, Akpolat N*** Kuş I*

*Department of Anatomy, Faculty of Medicine, Balıkesir University, Balıkesir, Turkey; **Health Collage, Mehmet Akif Ersoy University, Burdur, Turkey; ***Department of Pathology, Faculty of Medicine, Inönü University, Malatya, Turkey

Cadmium (Cd) is one of the heavy metals which widely used in industry and adversely affecting human health. The potential for protective effects of melatonin on cadmium-induced acute liver injury in rats was investigated in this work. Twenty-one male Wistar-Albino rats were randomly divided into three equal groups: Group 1; control, Group 2; cadmium chloride $(\mathrm{CdCl} 2)$, Group 3; cadmium and melatonin (Cd+Melatonin). Group 1 rats were injected salin. Group 2 rats were injected subcutaneously with $\mathrm{Cd}(1 \mathrm{ml} / \mathrm{kg})$ for 30 days. Group 3 rats were injected subcutaneously with $\mathrm{Cd}(1 \mathrm{mg} / \mathrm{kg})$ and injected intraperitoneally plus melatonin $(25 \mathrm{mg} / \mathrm{kg})$ for 30 days. All rats are killed by using decapitation method at the end of week experiment period. Hepar tissues were stained with heat shock protein 70 (Hsp70) immunohistochemically performed. Dense staining indicating Hsp70 immunoreaction was observed in the liver depending on the toxicity of cadmium in the rats of cad- mium group. The liver tissue sections of rats injected with melatonin and Cd, the HSP70 staining was detected minimal. It was detected that Melatonin hormone significantly reduced Hsp70 immunoreactions which is an indicator of cadmium toxicity and plays a protective role against the liver damage.

Keywords: Cadmium, melatonin, liver, Hsp70

\section{P-18}

\section{Immunohistochemical investigation of the effect of hCG treatment in obese rat adipose tissue}

Tunç $E^{*}$, Çalgüner E**, Erdoğan $\mathrm{D}^{* * *}$, Elmas $\mathrm{C}^{* * *}$, Öktem H*, Göktaş $\mathrm{G}^{* * *}$, Gözil R**, Bahçelioğlu $\mathrm{M}^{* *}$

${ }^{*}$ Researcher, Istanbul, Turkey; **Department of Anatomy, Faculty of Medicine, Gazi University, Ankara, Turkey; ${ }^{* *}$ Department of Histology and Embryology, Faculty of Medicine, Gazi University, Ankara, Turkey

Obesity is a clinical symptom due to the dysfunction of the hypothalamic center and the diet alone is not enough to control the weight. A novel treatment using daily injections of chorionic gonadotropin hormone (hCG) subcutaneously in anterior abdominal wall for loosing weight with well being of the patient is a current and attractive approach to obesity. Our aim was to study the immunohistochemical changes of the rat nearby and distant adipose tissue to the injection area those fed with cafeteria diet and then injected with low dose of subcutaneous hCG to investigate possible effects of this treatment. In this study, 20 Wistar albino rats, divided into two groups (obese and non-obese) were used. In each group, 7 rats received a single hCG injection while 3 rats were injected with saline solution as placebo during 5 week ( 5 day in each week). At the end of the five-week, all the animals were anaesthetized and sacrificed. Tissue samples from nearby and distant regions from the side of injection were obtained. Sections for immunohistochemical examination of ER- $\alpha, \mathrm{ER}-\beta$ and Leptin were done. Slides were examined with Photo-light microscope. In the obese group, morphological changes were observed in adipose cells which were thought to be the consequences of the obesity while after the hCG injections, cells with fused membranes were seen. This could be an effect of hCG, which cause to the release of intracellular contents. Leptin immunoreactivity was increase in obese hCG injected nearby adipose tissue while no changes were observed in the distant adipose tissue to the injection side. Such finding could suggest that the Leptin has only a local effect. We demonstrated that the hCG injections administered along with the obesity diet do not have any supplementary positive effect on rat adipose tissue.

Keywords: Adipose tissue, hCG, rat, immunohistochemistry

\section{P-19}

Morphometric and topographic features of the axillary nerve in fetal period

Uluer T*, Buluklu S**, Karşıyaka D**, Can E**, Aktekin $M^{* * *}$

*Institute of Health Sciences, Mersin, Turkey, **Faculty of Medicine, Mersin University, Mersin, Turkey; $* * *$ Department of Anatomy, Faculty of Medicine, Mersin University, Mersin, Turkey 
Main objective of this study is to determine the morphometric features of the axillary nerve along with its course and relationships with the accompanying structures particularly in the late period of fetal life. This will provide proper data about the axillary nerve to use during surgical and anesthetic procedures in early childhood. Axillary regions and the lateral axillary spaces of 17 fetuses (ages between 25-38 week-old) were bilaterally dissected under surgical microscope. Course and branches of the axillary nerve were determined. Length of the axillary nerve from its diverging point from the posterior fasciculus till the point where it gives its first branch was also measured. Distances between the nerve and the coracoid process and acromion during its course were measured. Its course beneath the deltoid muscle was determined. All measurements were performed with digital caliper. The thickness of the axillary nerve where it diverges from the posterior trunk was measured $1.33 \pm 0.41 \mathrm{~mm}$ on the right and $1.37 \pm 0.49 \mathrm{~mm}$ on the left. It was detected that the axillary nerve gives it first branch $14.49 \pm 3.98 \mathrm{~mm}$ and $14.64 \pm 3.98 \mathrm{~mm}$ after diverging from the posterior trunk on the right and left, respectively. The distance from the coracoid tip to the point where the nerve diverges from the posterior trunk was measured $16.19 \pm 4.16$ $\mathrm{mm}$ on the right and $16.69 \pm 3.93 \mathrm{~mm}$ on the left. The distance from the coracoid tip to the point where the nerve crosses the lateral border of the subscapularis muscle was measured $17.01 \pm 4.47$ $\mathrm{mm}$ on the right and $16.37 \pm 4.29 \mathrm{~mm}$ on the left. The distance from the posterior end of acromion to the point where the nerve emerges from the quadrilateral space was measured $13.98 \pm 2.29$ $\mathrm{mm}$ on the right and $14.54 \pm 2.33 \mathrm{~mm}$ on the left. After the anterior and posterior raphe dissections of the deltoid muscle, vertical distance between the nerve and the anterior end of acromion was measured $13.32 \pm 2.67 \mathrm{~mm}$ on the right and $13.74 \pm 3.11 \mathrm{~mm}$ on the left while the vertical distance between the nerve and the posterior end of acromion was measured $14.82 \pm 3.55 \mathrm{~mm}$ on the right and $15.63 \pm 2.47 \mathrm{~mm}$ on the left. The nerve was passing $2.93 \mathrm{~mm}$ and $2.76 \mathrm{~mm}$ under the glenoid labrum on the right and left, respectively. The course of the axillary nerve, the distance between this nerve and certain reference points and its thickness at several regions would be of use in certain surgical approaches such as humeral head surgery as well as in some procedures such as axillary biopsy or blockade and even in injections into the deltoid muscle, particularly in the very early postnatal period.

Keywords: Axillary nerve, lateral axillary space, fetus, axilla

\section{P-20}

\section{Morphological analysis of neovascularisation of sciatic nerve after appliying different sciatic nerve injury models}

Demirel BM *, Satı L **, Hızay A *, Tanrı̈̈ver G **, Özsoy U*, Öztürk S **, Sarıkcioğlu L *, Demir N **, Oğuz N*

*Department of Anatomy, Faculty of Medicine, Akdeniz University, Antalya, Turkey; **Department of Histology and Embryology, Faculty of Medicine, Akdeniz University, Antalya, Turkey

The aim of the study was to produce different injury models on sciatic nerve and to examine the effects of different injury mod- els from morphological point of view on neovascularisation of sciatic nerve. A total number of 110 female rats were used. In addition to control and sham groups, the rest of animals were divided into 3 different experimental groups: crush, total cut and hemisection. Experimental groups were examined on postoperative 48th hour, 14 th day and 1 st month. The sciatic nerve was crushed 30 seconds by a clamp. In total cut groups, transvers sectioning and then coaptation was performed. In hemisection group, the sciatic nerve was cut transversly along the half of the width. All injury zones were photographed before and after the operation. According to our results, various morphological differences were observed on sciatic nerve neovascularisation according on the nerve injury model. Neovascularization was observed both at site of the injury and at proximal side of the injury zone. Neovascularization was observed more organized in the hemisection groups; on the contrary neovascularzization was poorly organized in the other groups. Our study is of importance to reveal the effects of different injury models on sciatic nerve. Therefore, our data may yield a very important feature of peripheral nerve injury models and shed light to further studies in this field. We think that organized neovascularization of the sciatic nerve as observed in the hemisection groups may play an important role during regeneration.

Keywords: Sciatic nerve injury, crush, full cut, half cut, rat

\section{P-21}

\section{Examination of VEGF expression on crush injury model of the sciatic nerve}

Demirel BM*, Soygür B**, Satı GL ${ }^{* *}$, Hızay A**,

Tanrı̈̈ver $G^{* *}$, Özsoy $U^{* * *}$, Öztürk $S^{* *}$, Sarıkcığlu L**, Demir $\mathrm{N}^{* *}$, Oğuz $\mathrm{N}^{* * *}$

*Department of Anatomy, Faculty of Medicine, Ordu University, Ordu, Turkey; ${ }^{* *}$ Department of Histology and Embryology, Faculty of Medicine, Akdeniz University, Antalya, Turkey; ${ }^{* *}$ Department of Anatomy, Faculty of Medicine, Akdeniz University, Antalya, Turkey

Every year many people are affected by peripheral nerve injuries that results in undesirable social and economical lost. In order to overcome those lost to minimum, injuries must be treated as soon as possible. Sciatic nerve is commonly used to apply peripheral nerve injury models. Neuroscientists also investigate many factors affecting the regeneration process. Vascular endothelial growth factor (VEGF) is also a neurotrophic factor having a role not only in angiogenesis but also have effects on nervous system. In our study we aimed to investigate VEGF expression on rat sciatic nerve using crush injury models. 50 female rats were used. Rats were divided into crush control and sham main groups. Crush group also divided into subgroups as 48th hour, 15th day and 30th day. As determined in the schedule rats were sacrificed and sciatic nerve samples were collected for immunohistochemistry and western blot analysis. Immunohistochemically the most VEGF expression was seen in 48th hour subgroup. On the contrary the least expression was observed in 30th day subgroup. Western blot analysis revealed out similar results to immunohistochemical 


\section{S132 14th National Congress of Anatomy, 28 June-1 July 2012, Ankara, Turkey}

findings. The most and the least VEGF expression was seen in 30th days and 14th day subgroups respectively. We think that our results will help neuroscientists in the perspective of expressional way of VEGF in order to understand the degeneration and regeneration process during the peripheral nerve injury on different injury models of rat sciatic nerve and will shed light for further studies about the subject.

Keywords: Sciatic nerve, VEGF, crush injury, rat

\section{P-22}

The protective effects of proanthocyanidin and vitamin E againts damage caused by formaldehyde in the testes tissues of rat

Uluçam $\mathrm{E}^{*}$, Bakar $\mathrm{E}^{* *}$

*Department of Anatomy, Faculty of Medicine, Trakya University, Edirne, Turkey; ${ }^{* *}$ Department of Molecular Biology, Faculty of Science, Trakya University, Edirne, Turkey

This study investigated the protective effects of proanthocyanidin (PAC) and vitamin E (Evit) against formaldehyde-induced lipid peroxidation damage and morphological changes in rat testes. 28 male Wistar albino rats were divided into four groups. Group A was used as a control. Group B (FA) was injected every other day with formaldehyde for 14 days. Group $\mathrm{C}(\mathrm{FA}+\mathrm{PAC})$ was injected every other day with formaldehyde and bred everyday PAC for 14 days. Group D (FA+Evit) was injected every other day with formaldehyde and bred everyday Evit for 14 days. At the end of the experimental period animals were sacrificed and the testes removed. In addition, the levels of sialic acid (SA), malondialdehyde (MDA) and serum testosterone were determined. The serum and tissue levels of SA decreased significantly, the level of serum MDA significantly increased in animals treated with formaldehyde compared with the controls. Testosterone and tissue MDA levels were not found to be significant changes. Several necrotic changes were observed in testes tissues by light and electron microscopy. It was determinate that disordering of epithelia of seminiferous tubules, vacuolization between germinal epithelium cells, separated basement membrane were observed. Ultrastructurally, formaldehyde also caused disorganization and loss of mitochondrial cristae, dilatation in endoplasmic reticulum in testis tissues. The results suggest that formaldehyde has toxic effects in rat testis tissue. Vitamin $\mathrm{E}$ and proanthocyanidin have protective effect on formaldehyde toxicity in testis tissue.

Keywords: Testis, formaldehyde, proanthociyanidin, sialic acide, malondialdehyde

\section{P-23}

Evaluation of static postural control on young adults

Karadaş N, Uluçam E

Department of Anatomy, Faculty of Medicine, Trakya University, Edirne, Turkey

The purpose of this study, the data obtained from measurements of electronic devices during a quite stance is to determine the distribution among normal young adults and to contribute to creation of a normative database. Zebris FDM System Type FDM 1.5 is used in order to measure ground reaction force in the study included 60 healthy volunteer subjects between the age of 18- 24 ( 30 males and 30 females). Tests are performed base on four positions as feet together eyes open, feet together eyes closed, respectively right and left limb stance eyes open. When the eyes are closed, a significant increase on average path length value is observed on the each of the groups $(\mathrm{p}<0.05)$. There are statistically significant differences for both girls and boys in measurements except \%95 confidence ellipse angle between the positions of feet together eyes open and right limb stance eyes open $(\mathrm{p}<0.05)$. In the comparison between feet together eyes open and left limb stance eyes open, significant differences are found in average measurements except \% 95 confidence ellipse angle, Vertical Deviation values of boys and \%95 Confidence ellipse angle value of girls $(p<0.05)$. When the proprioception decreases, $\% 95$ confidence ellipse angle and vertical deviation values of boys are significantly larger on the right limb stance than on the left limb stance $(\mathrm{p}<0.05)$. Average Horizontal Deviation value of boys are larger than average horizontal deviation value of girls on both right and left limb stance, but while standing on the right foot, average vertical deviation value of girls is significantly larger than average vertical deviation value boys $(\mathrm{p}<0.05)$. As a result, some normative values for the static postural control of the young adults in the 18-24 age group of Turkish society were been obtained to make comparison in the pathologic situations later.

Keywords: Center of pressure, postural control, force platform, balance

\section{P-24}

For extension to femoral operation have done with unilateral dynamic fixator the importance of topographic anatomical landmarks in the region

\section{Cirpıcı O*, Cağimni $\mathrm{P}^{* *}$, Edizer $\mathrm{M}^{* * *}$}

*Department of Orthopedics and Traumatology, Özel Medicalpark Uşak Hospital, Uşak, Turkey; **Department of Anatomy, Faculty of Medicine, Afyon Kocatepe University Afyon, Turkey; ${ }^{* *}$ Department of Anatomy, Dokuz Eylül University, Faculty of Medicine, Izmir, Turkey

Limb lengthened operations are attempts to correction of limb inequalities occurring congenital, developmental and post-traumatic reasons. Surgical limb lengthening operations to increase success and reduce the risk of surgical complications, topographical anatomical features of the formations in the region are of importance for surgeons. 28-year-old male patient admitted to Medicalpark Usak Hospital because of post-traumatic pseudoarthrosis. The case, the normal length of $54 \mathrm{~cm} 5.5 \mathrm{~cm}$ shortening of the left femur as a result of the occurrence of anatomical structures taken into account during the operation evaluated retrospectively by means of $x$-ray identification, sugical significance of these structures determined the role of surgery relationship between each other and aimed at revealing the anatomical adaptation to prolong slow. During surgery, pre- 
serving periosteum and bone marrow aplied subtrochanteric corticotomy to the patient. After seven days operation, femur $0.25 \times 4 \mathrm{~mm} /$ day was extended by dynamic unilateral fixator. Totally femur was extended $53.5 \mathrm{~mm}$ (10 percent of the original femur length). In accordance with the literature, extension of $0.25 \times 4 \mathrm{~mm}$ per day; lengthening rate of all of the thigh muscle groups, the vascular and the neural structures of the region were equal with the femoral lengthening. During the operation, the periosteum and the bone marrow protection as a result of bone blood flow were observed in intact. By slow extension provided anatomical adaptation of the thigh was exposed. The anatomical markers were used to assess to protect the vascular circulation, the neural innervation and to prevent functional complications. Slow extension provided anatomical adaptation of the thigh was exposed. surgical complications was prevented with protection of the periosteum and bone marrow.

Keywords: Femur, dynamic fixator, lengthening operation, topographic anatomy, anatomical adaptation

\section{P-25}

\section{Evaluation of changes of anatomic and functional with nursing care in immobil events after cerebrovascular accident}

Aslan $\mathrm{N}^{*}$, Karabekir $\mathrm{H}^{* *}$, Göçmen Mas $\mathrm{N}^{* * *}$

*Karadeniz Technical University, Faculty of Medicine, Trabzon, Turkey; **Department of Neurosurgery, Faculty of Medicine, Kocatepe University, Afyonkarahisar, Turkey; ***Department of Anatomy, Faculty of Medicine, Dokuz Eylül University, Izmir, Turkey

This observational, cross sectional survey was conducted for multidisciplinary care planning and make them have quality life standart by proving anatomical, functional, sensory, and behavioral changes and deficiencies on individuals who has cerebrovascular disease diagnosis in Emergency Clinic of Karadeniz Technical University Farabi Hospital. 100 consecutive patients hospitalized were consisted the example of study in KTU Farabi Hospital Emergency Clinic at the date of JuneDecember 2011. At Statistical analysis of data percentage, arithmetic mean and chi square test was used and $\mathrm{p}<0.05$ significant was considered statistically. Descriptive form, applied to individuals receiving treatment in addition to the history of disease in $81 \%, 69 \%$ of HT, hemiparezi $93 \%$, 97\% of ataxia, dysarthria in $78 \%$, in $81 \%$ of bradykinesia were detected. 49 patients with right hemiplegia, lefthemiplegia in 48,3 patients had bilateral hemiplegia of totally 100 patients. In anatomical and radiological markers, 3 cases of bilateral hemiplegia, 2 patients were cross-paralysis. NIH, Karnofski, Barthel and Glasgow scales at the evaluation results were related to each other. In $89 \%$ of patients, moderate or severe or complete loss of sensation was observed. Hemisphere and the localization of hemiplegia were showed no significant effect on the $\mathrm{NIH}$ and the Glasgow coma scales. Our data researches were generally consistented with previous studies. For precise localization of pathology, it was found to be important the evaluation of radiologic anatomic datas. It has been reached the developing functional and anatomic changes highly affect the independence of patients.

Keywords: Anatomical changes, functional alterations, nursing care cerebrovascular accident

\section{P-26}

The results of students assesment about theorically and pratically anatomy session in Afyon Kocatepe University, 2009-2010

\author{
Lafçı $\mathrm{S}^{*}$, Göçmen Mas N** , Karateke $\mathrm{H}^{*}$, Taspınar C* \\ *Department of Anatomy, Faculty of Medicine, Afyon Kocatepe \\ University, Afyon, Turkey, **Department of Anatomy, Faculty of \\ Medicine, Dokuz Eylül University, İmir, Turkey
}

For medical students, the human body is the focus of investigation and intervention on a daily basis. For this reason, the study of anatomy in some form will continue to be essential to safe medical practice. It is necessary for core knowledge of anatomy to be assimilated by all doctors in order to practice and communicate safely. Medical education is regarded as a complex, demanding and often stressful procedure. Undergraduates, while enrolled in programmes of 6 years duration, are required to attain a unique and diverse collection of competences. Despite the major differences in anatomy educational systems, philosophies, methods and resources available worldwide, medical students' views regarding their education appear to be relatively convergent.Teaching methodology has been revolutionized with more reliance on models, imaging, simulation, and enhance the learning experience. In whole medical education anatomy has historically been a cornerstone. In recent decades wide-ranging changes have occurred in medical school curricula. Although anatomy curriculum is undergoing international reformation but the current framework lacks uniformity among institutions, therocally and pratically session are very important procedure for medical education Previous studies have established that medical students value their anatomy teaching during medical school. However, there is restricted data related to the written comments on student pleasure evaluations. This study was designed to evaluate these written comments. A questionnaire was prepared by the Anatomy Department of Medical School of the Afyon Kocatepe University and presented to 62 students in the beginning of the second semester to evaluate their thoughts about their anatomy education in the first and second semesters between 2009 and 2010 for every committee. This yielded a response rate of $80 \%$. We searched student pleasure on both medical anatomical practise and theoretical lectures. Overall nearly half of respondents believe they received insufficient anatomy teaching. The majority of medical students believed that it is feasible and beneficial to use cadavers and slides to teach gross anatomy. The preclinical teaching environments have shown the importance of developing symbiotic relationships between theorical and pratically session. In the near future, after rearragenment of the anatomical education and obtaining comparative results will be improved for learning advanced anatomical knowledge. And also we aimed to search student pleasure on anatomy teaching.

Keywords: Anatomy education 
S134 14th National Congress of Anatomy, 28 June-1 July 2012, Ankara, Turkey

\section{P-27}

\section{Morphometry of the hard palate and shapes of} transverse palatine sutura

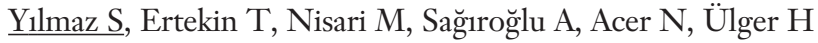
Department of Anatomy, Faculty of Medicine, Erciyes University Kayseri, Turkey

Hard palatal index values, which obtained by the measurements of hard palate are important for surgery and prosthetic dentistry. This research aims to measure morphometric values of hard palate and to calculate palatal index. In this research, we used 50 adult human dry skulls. We measured the length of median and transverse palatal sutura, and also measured the height, width and length of hard palate by a milimetric caliper. Palatal index (width/lengthx100) and palatal height index (height/width $\mathrm{x} 100$ ) were also calculated and transverse palatine sutura were classified with respect to their shapes. The relationships between the length of the transverse palatine sutura and the width of the cranium and also the median palatine sutura and the length of the cranium were examined. Palatal index values and palatal height index values were $77.62 \pm 8.14,23.11 \pm 8.68$, respectively. According to the palatal index values and palatal height index values we determined leptostaphyline palate $(60 \%)$ and chamestaphyline palate $(86 \%)$ respectively. In this study, the majority of the transverse sutures (32\%) were slightly smooth, transverse and similar shapes in the two sides. We found that there was no correlation between transverse sutura and with of skull, in addition same results was determined between median palatine sutura and length of skull ( $p>0.05)$. Knowledge of the anatomy of palate is clinically important for the surgical operations in this region. We think that our results may be contributed so far known.

Keywords: Hard palate, palatal index, transverse palatine sutura

\section{P-28}

Anatomic correlation of the entrapment of the superficial branch of the radial nerve

Gürses İA, Gayretli O, Kale A, Coşkun O, Usta A,

Şahinoğlu K, Öztürk A

Department of Anatomy, Istanbul Faculty of Medicine, istanbul University, istanbul, Turkey

Entrapment of the superficial branch of the radial nerve (SBRN), or Wartenberg's Syndrome, is a rare peripheral neuropathy with no motor component. The entrapment may result from radius fractures, lacerations, sustained pressure or may be iatrogenic. 5 cadavers were studied. The location of the SBRN and its branches were measured relative to the radial styloid (RS) and the dorsal tubercule of radius (DTR). The relation between the SBRN and the radial artery (RA), cephalic vein $(\mathrm{CV})$ and 1 st extensor compartment (1EC) was investigated. The forearms in the study were $229.2 \mathrm{~mm}$ (215.4-242 $\mathrm{mm})$ long. The SBRN emerged at a distance of $90,5 \mathrm{~mm}(81.6-$
98.,5 mm) and gave its first branch at a distance of $53.3 \mathrm{~mm}$ $(26.2-72.1 \mathrm{~mm})$ proximal to the RS. Then the SBRN passed $13.7 \mathrm{~mm}(5.1-23.4 \mathrm{~mm})$ lateral to the DTR. In all cadavers the SBRN or its branches were in close relation $(<2 \mathrm{~mm})$ with the $\mathrm{CV}$. The SBRN and its first branch were located at a mean distance of $8.7 \mathrm{~mm}(6-10.2 \mathrm{~mm})$ and $7 ., 8 \mathrm{~mm}(4.5-13.9 \mathrm{~mm})$ from the RA respectively. On 5 sides the SBRN and its first branch traversed the $1 \mathrm{EC}$ together, while on the remaining 5 only the first branch traversed the 1EC. On 1 side the SBNR pierced the brachioradialis tendon to become superficial. Our preliminary study showed that the reasons for the entrapment of SBRN are mostly based on its anatomy and new studies must be planned to investigate its relation with adjacent tendons.

Keywords: Superficial branch of the radial nerve, entrapment, Wartenberg's Syndrome

\section{P-29}

The comparison of the rom values of knee joint between obese and normal weight women

C.1kmaz S*, Çetkin $M^{* *}$

*Department of Anatomy, Faculty of Medicine, Trakya University, Edirne, Turkey; **Department of Anatomy, Instute of Health Science, Gaziantep University, Gaziantep, Turkey

It's important to evaluate the human motion and functions in explaining many pathological cases and developing new treatment methods. Therefore various tests and evaluation methods have been developed. With this idea, we aimed at comparing the Range of motion (Rom) values of knee joint in obese and normal weight women. 20 obese women in the average age of 30.6, and 20 normal weight women in the average age of 25.7 took part in our study. By their body mass index values, we determined if the participants were obese or not. We determined the individuals as obese who have $\geq 30 \mathrm{~kg} / \mathrm{m}^{2}$ body mass index and as normal who have $18.5-24.9 \mathrm{~kg} / \mathrm{m} 2$ body mass index. We measured the Rom values of knee joint by using Cybex electronic digital inclinometer. We measured flexion, extension, internal and external rotation movements. We found the average and standard deviation of Rom values of flexion, extension, internal and external rotation in obese and normal weight women respectively: $116.9^{\circ} \pm 6.7$ $-129.2^{0} \pm 4.3 ; 2.6^{0} \pm 1.2-1.9^{0} \pm 1.1 ; 11.6^{0} \pm 2.3-11.9^{0} \pm 1.9 ; 20.9^{0} \pm 3.8-$ $20.80 \pm 2.7$. In conclusion, increased body mass index in women limits the Rom values of flexion.

Keywords: Range of motion, knee joint, obesity

\section{P-30}

Investigation of mineral content of malleus and incus at scanning electron microscopy

Topal F*, Ünür E**, Yilmaz $\mathrm{S}^{* *}$, Nalbant $\mathrm{A}^{*}$

*Department of Anatomy, Faculty of Medicine, Eskişehir Osmangazi University, Eskişehir, Turkey; **Department of Anatomy, Faculty of Medicine, Erciyes University, Kayseri, Turkey 
There are three mobile ossicles at the middle ear cavity between eardrum and inner ear. Among these ossicles; the malleus articulates with the body of incus, the stapes articulates with the lenticular process of incus and the last one, the incus articulates with both the malleus and the stapes. Our study was performed on 10 malleuses and 10 incuses. These ossicles were examined at scanning electron microscopy and then 6 areas were determined to analyze mineral content of these ossicles. While one of the determined areas was outside of the pathway, transmitting the sound resonance to the inner ear, the other 5 areas were on the pathway of sound vibrations. Total mineral content (calcium, phosphorus, magnesium, sodium, carbon and oxygen) were $\% 30.94, \% 15.43, \% 0.23, \% 0.42, \% 13.97$, and $\% 38.82$ for the malleus; \%30.29, \% 15.45 , \%0.25, \%0.38, $\% 13.93$, and $\% 39.42$ for the incus, respectively. The mineral analysis of determined 6 areas was compared statistically. The calcium and phosphorus contents of the malleus and the incus were found significantly different between the parts of the pathway that are transmitting or not transmitting the sound resonance to the inner ear $(\mathrm{p}=0.05)$.

Keywords: Malleus, incus, mineral, scanning electron microscopy

\section{P-31}

\section{Objective analysis of singing voice}

Berdan M, Yücel AH, Petekkaya E

Department of Anatomy, Faculty of Medicine, Çukurova University, Adana, Turkey

Lateral thyrohyoid ligament is located between hyoid bone and superior corn of thyroid cartilage of larynx. At puberty, larynx enlarges rapidly, in boys. Because of extension of vocal cords their voice change and become hoarse. In girls, larynx enlarges more slowly. In this reason, their voices don't change. Sixteen males and 16 females were enrolled in this study. If the subjects don't have voice training, they are classified as a Control Group. If the subjects have start voice training recently, in Department of Opera and Chant, State Conservatory of Cukurova University (S.C.C.U.), they were classified as a Group I. The subjects in Group II have had voice training in Department of Opera and Chant, S.C.C.U. and stil continuing their training. Right and left lateral thyrohyoid ligament were measured by palpation, in all groups. In order to show the accuracy of the ligament measurements, laryngeal neck graphy of the participants have been obtained, imaged and remeasured by Magnetic Resonance (MR). Voice analyses of the three groups (jitter, shimmer and fundamental frequency) are also performed. The measurements were performed considering those in Group I who have recently started the education and at the end of the eight month education period reperformed in all three groups. The measurements were assessed statistically. Keywords: Hyoid bone, lateral thyrohyoid ligament, vocal cords, voice
P-32

Whole-body vibration exercise improves body weight support and bladder function in spinal cord-injured rats

Wirth $\mathrm{F}^{*}$, Schempf $\mathrm{G}^{*}$, Stein $\mathrm{G}^{* *}$, Ankerne $\mathrm{J}^{*}$, Ashrafi $\mathrm{M}^{*}$, Semler $\mathrm{O}^{* * *}$, Angelova $\mathrm{S}^{* * * *}$, Eisel L*, Harrach $\mathrm{R}^{*}$, Özsoy $\mathrm{O}^{* * * * *}$, Özsoy $\mathrm{U}^{* * * * * *}$, Schonau $\mathrm{E}^{* * *}$, Angelov DN*, Irintchev $\mathrm{A}^{* * * * * * *}$

Department of Anatomy, Koln University, Koln, Germany; **Department of Orthopedics and Traumatology, Koln University, Koln, Germany; $* * *$ Child Hospital, Koln University, Koln, Germany; ****Jean-Uhrmacher Institute of ENT, Koln University, Koln, Germany; $* * * * *$ Department of Physiology, Akdeniz University, Antalya, Turkey; $* * * * * *$ Department of Anatomy, Faculty of Medicine, Akdeniz University, Antalya, Turkey; $* * * * * * *$ Department of ENT, Jena University, Jena, Germany

Whole-body vibration (WBV) is a relatively novel form of exercise used to improve neuromuscular performance in healthy individuals. Its usefulness as a therapy for patients with neurological disorders, in particular spinal cord injury (SCI), has been barely explored in clinical settings and not tested in animal models of SCI. We performed severe compression SCI at low-thoracic level in adult female Wistar rats and subjected them to daily WBV training starting 7 or 14 days after injury (WBV7 and WBV14, respectively) and continuing over a 12week post-injury period. Rats with SCI but no WBV training (sham) served as controls. Recovery of locomotion was analyzed using video recordings of beam walking and inclined ladder climbing. The functional status of the bladder was assessed immediately prior to each manual voiding i.e. 3 times daily, using an own scoring method. Locomotor rating and numerical assessment of plantar stepping and skilled limb movements revealed no significant effects of $\mathrm{WBV}$ versus sham treatment during the observation time period. However, compared with sham-treated rats, WBV14 but not WBV7 significantly improved body weight support during ground locomotion and overall recovery by 6-12 weeks after SCI. Most remarkably, WBV14 lead to a significant improvement of bladder function at 6-12 weeks after injury. These findings provide first evidence for positive functional effects of WBV in an animal SCI model and warrant further preclinical investigations on $W B V$ as a potential rehabilitation therapy for neurological disorders.

Keywords: Spinal cord injury, nerve regeneration, whole body vibration, rehabilitation

\section{P-33}

Age related changes in height and shape of the lumbar intervertebral discus

Malkoç İ*, Aydınlıoğlu S**, Alper F*** Kaçıroğlu F*, Yüksel $\mathrm{Y}^{* * * *}$, Yüksel $\mathrm{R}^{* * * * *}$, Kara $\mathrm{A}^{*}$, Dane $\mathrm{S}^{* * * *}$, Toktaş $\mathrm{M}^{* * * * * *}$

*Department of Anatomy, Faculty of Medicine, Atatürk University, Erzurum, Turkey; **Department of Anatomy, Faculty of Medicine, Yüzüncü Yıl University, Van, Turkey; ***Department of Radiology, Faculty of Medicine, Atatürk University, Erzurum, Turkey; $* * * *$ Department of Histology and Embryology, Faculty of Medicine, Fatih University, Ankara, Turkey; $* * * * *$ Department of Physiology, Faculty of Medicine, Fatih University, Ankara, Turkey; $* * * * * *$ Department of Anatomy, Faculty of Medicine, Fatih University, Ankara, Turkey 


\section{S136 14th National Congress of Anatomy, 28 June-1 July 2012, Ankara, Turkey}

There was no agreement on intervertebral disc changes by age in human lumbar spine in the previous studies. The present study aimed the re-examination of the possible changes in intervertebral lumbar discs by age in healthy subjects. Normal lumbar spine magnetic resonance imaging (MRI) scans were obtained from 171 individuals ( 84 men, 87 women; $12-80$ years old). The anterior and posterior heights and the depths of the intervertebral discs at each level of the lumbar spine were measured. The gender and age-related changes were not significant for heights. The depths of the intervertebral discs changed with age significantly in both sex. The average disc height and shape index increased with age significantly in some disks individually. The disk depths only showed gender difference. The present study did not find clear increases in all dimensions and the shape of the discs of both sexes with age and differed from the previous studies. Our findings needed to be confirmed by the further MRI studies.

Keywords: Intervertebral disc, morphometry, radiology, age

\section{P-34}

A case with muscular, arterial and nervous variations in the upper limb

Çatlı $\mathrm{MM}^{*}$, Özsoy U**, Kaya $\mathrm{Y}^{* *}$, Hizay A**, Yıldırım FB ${ }^{* *}$, Sarıkcioğlu L**

*Faculty of Medicine, Akdeniz University, Antalya, Turkey; Faculty of Medicine, **Department of Anatomy, Akdeniz University, Antalya, Turkey

Co-existence of variations in the upper limb is rare. In this study we aimed to describe multiple variations in the upper limb and discuss their co-existence from clinical and embryological points of view. During a routine dissection studies, we found multiple variations on the left side of a 51-year-old Turkish male cadaver. The arm was dissected carefully to display all structures. All other related structures were also exposed. Four-headed biceps brachii muscle, three-headed coracobrachialis muscle, high originated radial artery and communication between median and musculocutaneous nerves were encountered. We think that such co-existence should be kept in mind in surgical and diagnostic procedures in the upper limb.

Keywords: Multiple variations, biceps brachii muscle, coracobrachialis muscle, radial artery, musculocutaneous nerve, median nerve

\section{P-35}

\section{Role of the vasa nervorum on nourishment of the sciatic nerve}

Sarıkcığlu L*, Demirel BM*, Yıldırım FB*, Hızay A*, Demir $\mathrm{N}^{* * * * *}$, Oğuz $\mathrm{N}^{*}$

${ }^{*}$ Department of Anatomy, Faculty of Medicine, Akdeniz University, Antalya, Turkey; **Department of Histology and Embryology, Faculty of Medicine, Akdeniz University, Antalya, Turkey; ${ }^{* \star *}$ Electron Microscopy Unit, Faculty of Medicine, Akdeniz University, Antalya, Turkey
Vasa nervorum are well-developed collateral vascular system and form intraneurial and extraneurial plexi. These vessels originate mainly from the popliteal and inferior gluteal arteries. In the present study we aimed to study the relation between epineurial vessels and internal arterial plexus. A total number of 40 male rats were used for this study. The animals were divided into four groups (Control, Sham, Group 1, Group 2). In Group 1, epineurial vessels contributing to the formation of the vasa nervorum of the sciatic nerve were ligated from their origination (from inferior gluteal and popliteal arteries) to induce anoxia. In group 2, the vasa nervorum around the sciatic nerve was stripped by taking care not to damage the epineurium. Analysis of the sciatic nerve samples revealed oedematous appearance in Group 1. However, in Group 2, subperineurial degeneration/demyelinization was observed. Ligation of the epineurial vessels from their origination did not create an ischemic fiber degeneration. This anoxia was compensated by blood supply from internal arterial plexus. We think that this study would be helpful to studies on simulation of the vasculitic neuropathy.

Keywords: Sciatic nerve, epineurial vessels, vasa nervorum

\section{P-36}

Anthropometric measurements of infraorbitale foramen

Nisari M, Çınar S, Ertekin T, Aycan K, Unur E

Department of Anatomy, Faculty of Medicine Erciyes University, Kayseri, Turkey

The groove is called infraorbital groove that continues infraorbital channel in maxillar bone. This channel which transmits the infraorbital vessels and nerve is opened by infraorbital foramen the front of maxilla by infraorbital foramen, In this study we aimed to determine the rate of infraorbital foramen (notch) on skulls and to find out the distances between this foramen and anatomic landmarks. This research was studied on 50 human dry skulls in Erciyes University in Faculty of Medicine Department of Anatomy Laboratuary. In this study seventeen morphometric measurements related to the distance between the infraaorbital foramen to orbit wall and the other reference points were determined in bone using a millimetric compass. The mean distance between the right infraorbital foramen and left infraorbital foramen was measured as $5.54 \pm 0.38 \mathrm{~cm}$. The mean distance between the infraorbital foramen and superior margin was measured as $4.12 \pm 0.27 \mathrm{~cm}$ on the right side and $4.17 \pm 0.29 \mathrm{~cm}$ on the left side. The mean distance between the infraorbital foramen and inferior margin was measured as $0,71 \pm 0,18 \mathrm{~cm}$ on the right side and $0.72 \pm 0.20 \mathrm{~cm}$ on the left side. The distance between the infraorbital foramen and the other anthropometric points were not showed significant difference according to sides. The localization of infraorbital foramen is important in during local anaesthesia planning, maxillofacial and orbital surgery. It is necessary to know this anatomic area for avoiding to damage infraorbital vessels and nerve. In this study, we tried to determine the morphologic and morphometric anatomy of infraorbital foramen.

Keywords: Infraorbital foramen, anthropometry, morphometry 


\section{P-37}

Interparietal bone (Inca bone) in adult human skull

Özçelik O, Yılmaz S, Arslan A, Nisari M, Ertekin T, Unur E, Aycan K

Department of Anatomy, Faculty of Medicine, Erciyes University, Kayseri, Turkey

The squamous part of occipital bone consists of two parts, supraoccipital and interparietal. The interparietal portion ossifies intramembranously and in rare cases may be separated from the supraoccipital part by a suture. It is than called as the interparietal or inca bone. The occurrence of inca variable is rare in humans. In this study we aimed to investigate frequency of os incae in the human skulls and determine distances between surrounding structures and os incae. The material used in this study consisted of 80 skulls in the Department of Anatomy laboratory, Medical Faculty of Erciyes University. We measured distances between centrum of os incae and various anatomic land marks. In addition we measured transvers and vertical diameter of os incae. We examined 80 skulls and we found 7 ince bones $(8.7$ percent). Six of them consist of single part and one of them consists of three parts. We measured that mean transvers and sagittal diameters of six bones were $4.15 \pm 1.02 \mathrm{~cm}$ and $3.13 \pm 1.03 \mathrm{~cm}$, respectively. Knowledge of inca ossicles in human skulls may be useful to neurosurgeons, radiologists and anatomists for clinical studies and antropologic studies.

Keywords: Inca bone, human skull, morphometry

\section{P-38}

Effect of endothelin-A receptor antagonist BQ 123 on sciatic nerve injury in rats

Kasap Z, Çakıl D, Ekici F, Erdoğan H, İnanır A, Mumcuoğlu İ Faculty of Health Sciences, Gaziosmanpaşa University Tokat, Turkey

Periferic nerve injuries are diseases that cause problems with physical and socio-economical restriction. Endothelins are known to have potent vasoconstructive, mitogenic and also increasing effect on collagen synthesis. Endothelin A (ET-A) receptor antagonists are thought to have a potential on treatment of sciatic nerve injury because of their positive effects directly on nerve tissue and also tissue perfusion; so the effects of ET-A receptor antagonist, BQ 123 on sciatic nerve injury were investigated in this study. $21 \mathrm{Wistar}$ albino male rats were used throughout the study and were divided to 3 groups as follows; control, sciatic nerve injury and sciatic nerve injury + BQ 123 treatment groups, each consisting of 7 rats. The sciatic nerve injury was generated by compression for 3 minutes with a clamp performing 54 Newtons pressure. The treatment group was administrated BQ 123 ( $1 \mathrm{mg} / \mathrm{kg} /$ day; intravenous) for 3 days, the first dose soon after the injury. All rats were performed the walking path analysis in 7, 14, 21 and 30th days after the injury and their sciatic function indexes (SFI) were calculated after each analysis. Also electrophysiological recordings (EMG) were performed two times; the first was in the acute phase, soon after the injury and the second was in the 30 th day after the injury. After the compression performed with the clamp, it was observed that the nerves were damaged but their continuity was conserved. Complete flask paralysis was occurred in all extremities damaged after surgery. The improvement in both SFI and EMG values were greater in BQ 123 administrated group after the injury, compared to nerve injury administrated group. BQ 123 shows neuroprotective effects on sciatic nerve injury formed by compression.

Keywords: Sciatic nerve injury, endothelin

\section{P-39}

Effects of ginkgo biloba on penicilin-induced epileptiform activity in rats

Çakıl D*, Kasap Z ${ }^{* *}$, Kozan R*** Aygün $\mathrm{H}^{*}$

*Department of Anatomy, Faculty of Medicine, Gaziosmanpaşa University, Tokat, Turkey; **Health High School, Gaziosmanpaşa University, Tokat, Turkey, $* * *$ Department of Physiology, Faculty of Medicine, Bezmialem Foundation University, Istanbul, Turkey

Free radical and oxidant enzyme generations are involved in mechanisms of epilepsy. The role of Ginkgo biloba extract (EGB 761 ) in treatment of diseases caused by free radicals and oxidative damage has been investigated in many studies. Aim of the present study was to clarify the effects of EGB 761 on penicilin induced epileptiform activity in rats. $28 \mathrm{Wistar}$ albino male rats were divided into 4 grups as 1 control and 3 experimental. Left somatomotor cortex was exposed by craniotomy and ball electrodes were placed over the cortex. Epileptic focus was produced by penicillin $\mathrm{G}(500 \mathrm{IU})$ injection. The experimental groups were administrated EGB 761, $30 \mathrm{~min}$ after penicilin injection at 50 $\mathrm{mg} / \mathrm{kg}, 100 \mathrm{mg} / \mathrm{kg}$ and $150 \mathrm{mg} / \mathrm{kg}$ doses, respectively. The ECoG activity was monitored for 2 hours. Epileptiform activity was formed on ECoG recording of all animals in 1-3 minutes after penicillin G injection. EGB 761 showed antiepileptiform effects at all administrated doses. The most effective dose was determined as $100 \mathrm{mg} / \mathrm{kg}$. EGB 761 generates a dose depended detractive effect on penicilin induced epileptiform activity in rats.

Keywords: Ginkgo biloba, penicilin induced epileptiform activity

\section{P-40}

\section{Congenital left ventricular diverticulum: report of two cases}

Koşar Mí* Karapınar $\mathrm{H}^{* *}$, Küçükdurmaz $\mathrm{Z}^{* *}$, Gül İ**, Sabancioğulları $\mathrm{V}^{*}$, Yilmaz $\mathrm{A}^{* *}$

*Faculty of Medicine, Department of Anatomy, Cumhuriyet University Sivas, Turkey; ${ }^{*}$ Department of Cardiology, Faculty of Medicine, Cumhuriyet University Sivas, Turkey**

Left ventricular diverticula are rarely seen congenital abnormalities. It has been reported that they are mostly located in apical zone of inferior and anterior wall. In this article, we present two diverticula facts located in interventricular septum. Case 1: A 54- 
S138 14th National Congress of Anatomy, 28 June-1 July 2012, Ankara, Turkey

year old woman complained about palpitation, and during the echocardiography applied, a tubular diverticulum structure in the length of $22 \mathrm{~mm}$ and width of $7 \mathrm{~mm}$ in the diastole located in mid zone of interventricular septum, of which lumen narrows while flexing in the systole, was observed. In 3-year follow-up of the fact which showed no arrhythmia during the examinations, no complication was found. Case 2: A 43 year-old woman complained about palpitation and chest pain. During the echocardiography applied, a left ventricular diverticulum in the length of 20 $\mathrm{mm}$ and width of $13 \mathrm{~mm}$ in the diastole located in apical zone of interventricular septum was observed. It was observed that the lumen was narrowing greatly in diverticulum systole. It was begun to follow up the fact which showed no additional pathology was observed during the examinations. Conclusion: Left ventricular diverticula occur with full protrusion of ventricular wall as a result of poor development of the myocardium in embryological life. They have two types which are fibrous and muscular. They are distinguished from aneurisms with their flexes in systole. In myocardial non-compaction which is the other congenital abnormality important in distinctive diagnosis, there are more than three myocardial recesses. As diverticula generally accompany to other cardiac defects and medium defects, they are diagnosed during childhood. It has been reported that they may lead to the complications such as arrhythmia, systemic emboli, heart failure, valvular deficiency, rupture and immediate death. However, most of them are diagnosed randomly.

Keywords: Congenital anomaly, ventricular diverticulum, echocardiography

\section{P-41}

Measuring the orbital volumes of orbitas with cellulitis by stereological method in computerized tomography

Erbağc1 H*, Okumuş $\mathrm{S}^{* *}$, Kervancioğlu $\mathrm{P}^{*}$, Kervancıŏglu $\mathrm{S}^{* * *}$, Kızılkan $\mathrm{N}^{*}$, Erbağgı I

*Department of Anatomy, Medical Faculty, Gaziantep University, Gaziantep, Turkey; **Department of Ophthalmology, Gaziantep University Medical Faculty, Gaziantep, Turkey; ***Department of Radiology, Gaziantep University Medical Faculty, Gaziantep, Turkey

Orbital cellulitis is the soft tissue infection behind the orbital septum. Our aim in this study is comparing the volumes of healthy orbitas and orbitas with cellulitis in CT, a procedure used in the diagnosis and follow up of orbital cellulitis, by Cavalieri method. The average age of the 16 patients ( 8 male, 8 female) enrolled in the study was 30.4. Computerized tomography (CT) images of the patients in the acute phase were evaluated. The volumes of healthy orbitas and orbitas with cellulitis were calculated and compared by the aid of a stereo investigator computer assisted a sterological analysis equipment that uses a special software programme. Seven patients had right orbita cellulitis while the remaining nine had a diagnosis of left orbital cellulitis. The volume of healthy orbita was found to be $32 \pm 14$ whereas the volume of orbitas with cellulitis was $40 \pm 12$. Bigger volumes of orbitas with cellulitis compared to healthy orbitas were statistically significant. The limitation of the movements of extraocular muscles and the presence of propitosis raises the suspicion of orbital cellulitis, however these findings have to be supported by CT findings. The authors believe that quantification of the orbital measurements of orbitas with cellulitis contributes to grading the infection, awareness of surgical indications and monitoring the results of the therapy.

Keywords: CT, orbital cellulitis, volume, stereology

\section{P-42}

\section{Estimation of the brain stem volume by stereological method on magnetic resonance imaging}

\section{Erbağc1 $\mathrm{H}^{*}$, Keser $\mathrm{M}^{*}$, Kervancıoğlu $\mathrm{S}^{* *}$, Kızılkan $\mathrm{N}^{*}$}

*Department of Anatomy Faculty of Medicine, Gaziantep University, Gaziantep, Turkey; **Department of Radiology, Faculty of Medicine, Gaziantep University, Gaziantep, Turkey

Neuron loss that occurs in some neurodegenerative diseases can lead to volume alterations by causing atrophy in the brain stem. The aim of this study was to determine the brain stem volume and the volume ratio of the brain stem to total brain volume related to gender and age using new stereo investigator system in normal subjects. MR images of 72 individuals who have no pathologic condition were evaluated. The total brain volumes of female and male were calculated as $838.52 \pm 68.2 \mathrm{~cm}^{3}, 930.24 \pm 98.9 \mathrm{~cm}^{3}$, respectively. Brain stem volumes of female and male were determined as $18.99 \pm 2.36 \mathrm{~cm}^{3}, 22.05 \pm 4.01 \mathrm{~cm}^{3}$, respectively. The ratios of brain stem volume to total brain volume were $1.96 \pm 0.17$ in female and in $2.05 \pm 0.29$ male. The total brain and brain stem volumes were observed smaller in female and it is statistically significant. Among the individuals whose ages are between 20 and 40 , total brain and brain stem volume measurements with aging were not statistically significant. We believe that the measurement of brain stem volume with an objective and efficient calculation method will contribute to the early diagnosis of neurodegenerative diseases, as well as to determine the rate of disease progression, and the outcomes of treatment.

Keywords: Brain stem, Cavalieri principle, volume, stereology

\section{P-43}

\section{Effects of quercetin, fish omega-3 fatty acids and melatonin on the changes in cerebrum in rats exposure to cadmium}
Akçer $\mathrm{S}^{*}$, Yağmurca $\mathrm{M}^{* *}$, Songür A*, Özen $\mathrm{OA}^{* * *}$, Mollaoğlu $M^{* * * *}$, Tosun $M^{* * * *}$, Alkoç OA******
*Department of Anatomy, Faculty of Medicine, Afyon Kocatepe University, Afyonkarahisar, Turkey; ${ }^{* *}$ Department of Histology and Embriology, Faculty of Medicine, Fatih University Ankara, Turkey; $* * *$ Department of Anatomy, Faculty of Medicine, Namık Kemal University, Tekidağ, Turkey; $* * * *$ Department of Physiology, Faculty of Medicine, Şifa University, Izmir, Turkey; *****Department of Histology and Embriology, Faculty of Medicine, Afyon Kocatepe University, Afyonkarahisar, Turkey; $* * * * * *$ Department of Anatomy, Faculty of Medicine, Düzce University, Düzce, Turkey 
Cadmium (Cd) is one of the toxic heavy metals. In our study, we investigated those toxic effects of $\mathrm{Cd}$ on rat's cerebrum and whether there are protective effects of quercetin, fish omega-3 fatty acids and melatonin because of their antioxidant properties. 52 wistar Albino male rats were divided into six groups: 1) Control (1 mg/kg/d-ip saline), 2) Cd (1 mg/kg/d, gavage,6 weeks), 3) Cd+Melatonin (25 mg/kg/d-ip), 4) Cd+Quercetin (270 mg/kg/d-gavage), 5) Cd+fish n-3 fatty acid $(400 \mathrm{mg} / \mathrm{kg} / \mathrm{d}-$ gavage), 6) Cd+ Quercetin+fish n-3 fatty acid. All rats were sacrificed under anesthesia at the end of the exposure period, cerebrums were removed. Left hemispheres of cerebrum from each rat were used for biochemical analysis and right hemispheres were used for morphological evaluation. After routine tissue preparation, cerebrum sections were stained with cresyl violet acetate. Activities of superoxide dismutase (SOD) and glutathione peroxidase (GSH-Px) and malondialdehyde (MDA) levels were determined. Light microscopic evaluation of the $\mathrm{Cd}$ group revealed a marked decrease in the number of cells in parietal cortex along with picnotic and hyperchromatic changes in the hippocampal region. SOD activity was found to be increased in Cd group except cerebral tissue. GSH-Px activity and MDA levels were increased in all tissues in Cd group. Morphological changes were treated largely with melatonin, quercetin and quercetin-fish n-3 fatty acid, but they were treated partially with only fish n-3 fatty acid. Increase in tissues MDA levels and SOD activity was decreased with granting together fish n-3 fatty acid and quercetin with melatonin. The increase in GSH-Px activity can be prevented granting together fish n-3 fatty acid and quercetin with melatonin. We concluded that Cd causes morphologic and biochemical injury in rat cerebrum; it may be partially prevented by giving antioxidant-acting quercetin, fish $n-3$ fatty acids and melatonin.

Keywords: Cadmium, cerebrum, melatonin, quercetin, fish $\mathrm{n}$ 3 fatty acids

\section{P-44}

\section{Sex determination from humerus by morphometric methods}

Metin TA*, Karakaş $\mathrm{S}^{* *}$

*Department of Anatomy, Faculty of Medicine, Adnan Menderes University, Aydın, Turkey, **Department of Histology Embryology, Faculty of Medicine, Adnan Menderes University, Aydın, Turkey

In this study on the different regions of the humerus with 22 sheets of morphometric measurements, to define in detail the morphological features of the humerus and aimed to the detection of gender-related differences. Research, 104 pieces (52 female, 52 male) in the left side of the humerus of adult. On Humerus; proximal eight, three on the trunk, the distal end 11, for a total of 22 measurements were performed. Measurements for the millimeter caliper and rigid tape measure were used for measurement of the humerus length, osteometric board. Obtained data were analyzed with SPSS (14.0 version) program. Compliance with all of the normal distribution of data Kolmogorov-Smirnov test was applied. Gender differences, Student's t-test for normally distributed variables for the normal undistorted variables were assessed with the Mann-Whitney U test. In addition, Fisher's linear discriminant analysis with the help of metric values between the sexes was found that the best separation. Different 22 measurements were made on humerus in which morphometric tests were performed to determine sex including eight on proximal end, three on shaft and 11 on distal end. Of 22 parameters measured, depth of Fossa radialis (DFR) and width of Trochlea humeri (WTH) were considered as insignificant based on test results. Other measurements were regarded significant. Humerus taken every measure was adopted as the only variable 'Fisher linear discriminant function test' applied, the most reliable variables, $89.4 \%$ 'luxury HBTC with the separation rate, TDC, was Min GC. Than all of the variables' Fisher linear discriminant function test 'applied to $98.1 \%$ of men and women $100 \%$ ' s correctly classified $99 \%$ of the whole group. Metric data obtained in our study shall be useful in case of that sex of skeleton could not be determined morphologically. Our study was cross-sectional and does not reflect entire Turkish society, but provides general knowledge. Therefore, we are opinion of that it could be referred in studies in which sex determination is made using humerus.

Keywords: Sex determination, discriminant analysis, morphometry, humerus

\section{P-45}

Morphometric evaluation of parasagittal venous anatomy for intracranial approaches: a cadaveric study

Sayhan $S^{*}$, Güvencer $M^{* *}$, Özer E*** Arda $M^{* * * *}$

*Department of Neurosurgery, Erpa Special Health Hospital, Denizli, Turkey, **Department of Anatomy, Faculty of Medicine, Dokuz Eylül University, Izmir, Turkey, ${ }^{* * *}$ Department of Neurosurgery, Faculty of Medicine, Dokuz Eylül University, Izmir, Turkey

Obstruction of superior sagittal sinus (SSS) and collateral briding veins is a well-known reason of postoperative brain edema and brain infarct, however, morphometric anatomic studies done in the light of surgical landmarks aren't sufficient in number. Object of this study is to describe venous structures releated to SSS with silicon injected cadaveric models. This study was on 6 silicon injected cadaveric heads at Anatomy Department. Duramater was removed and veins on parasagittal area were examined. SSS morphology, veins draining into SSS, their size, number and distance were evaluated. Mean vein number draining into SSS is $2.9 \pm 1.5$ at anterior to coronal suture (CS), between CS and vertex is $3.2 \pm 0.8$, between vertex and lambdoid suture (LS) is $2.3 \pm 0.9$, between LS and confluens sinium $0.3 \pm 0.5$. There was no statically difference between right and left sides $(\mathrm{p}=0.140, \mathrm{p}>0.05)$. Diameter of veins was $2.4 \pm 1.0 \mathrm{~mm}$ at anterior to $C S, 3.0 \pm 1.2 \mathrm{~mm}$ at between $\mathrm{CS}$ and vertex, $2.4 \pm 0.7 \mathrm{~mm}$ at between vertex and LS, and $2.2 \pm 0.5 \mathrm{~mm}$ at between LS and confluens sinium. Knowing details of anatomic structures of SSS and venous structures draining into it may protect the patients from many surgical complications. SSS and related structures with surgical landmarks are valuable for neurosurgeons.

Keywords: Superior sagittal sinus, parasagittal venous anatomy, intracranial superficial veins, silicon injected cadaver 


\section{P-46}

The protective effect of atropine on the fenthion caused morphology of the cerebellar Purkinje cell toxicity

Baş O*, Aslan A**, Söztanacı US*, Sönmez ÖF***,

Tümkaya $L^{* * * *}$, Yağmurca $M^{* * * * *}$, Toktaş $M^{* * * * *}$

*Department of Anatomy, Faculty of Medicine, Rize University, Rize, Turkey; **Department of Physiology, Faculty of Medicine, Rize University, Rize, Turkey; $* * *$ Department of Neurosurgery, Samsun Training and Research Hospital, Samsun, Turkey; $* * * *$ Department of Histology and Embryology, Faculty of Medicine, Rize University, Rize, Turkey; $* * * * *$ Department of Histology and Embryology, Faculty of Medicine, Fatih University, Ankara, Turkey; $* * * * * *$ Department of Anatomy, Faculty of Medicine, Fatih University, Ankara, Turkey

Fenthion is an organophosphate compound that is widely used throughout the world as an insecticide. The widespread availability of the organophosphate compounds causes millions of poisoning cases each year. So we aimed to investigate the toxic effect of fenthion on purkinje pell morphology in cerebellum using nucleator methods and to examinate whether atropine has protective effects. Eighteen female adult Wistar albino rats were used in study. Rats were randomly divided into three groups. Control group received a single dose of $0.8 \mathrm{~g} / \mathrm{kg}$ subcutaneous saline followed by $2 \mathrm{mg} / \mathrm{kg} / \mathrm{h}$ saline intraperitoneally for four hours. Fenthion group received single dose of $0.8 \mathrm{~g} / \mathrm{kg}$ subcutaneous fenthion followed by $2 \mathrm{mg} / \mathrm{kg} / \mathrm{h}$ saline intraperitoneally for four hours. Fenthion+atropine group received a single dose of $0.8 \mathrm{~g} / \mathrm{kg}$ subcutaneous fenthion followed by $2 \mathrm{mg} / \mathrm{kg} / \mathrm{h}$ atropine intraperitoneally for four hours. On the fifth day, animals were sacrificed and sequential sections of cerebellum were stained with Cresyl Fast Violet. Circumference and diamater of neurons were measured by nucleator method. The structure of pyramidal cells was normal in the control grup. Swelling, and pyknosis were observed in the cytoplasm of purkinje cells in Fenthion group. Swelling of the cells caused an increase in area, circumference and diamater changes of neurons. While there was no significant neuronal circumference and diamater changes in cerebellum in Fenthion+Atropine grup. Fenthion significantly, increases apoptosis, causes neuronal swelling and increase in area, circumference and diamater of neurons. Harmful effects of fenthion can be treated by atropine.

Keywords: Fenthion, atropine, stereology, cerebellum

\section{P-47}

Partial agenesis of corpus callosum: case report

Sağıroğlu A*, Coşkun A**, Açer N*, Ertekin T*, Yılmaz S*, Öcal ZÖ**

*Department of Anatomy, Faculty of Medicine, Erciyes University, Kayseri, Turkey; **Department of Radiology, Faculty of Medicine, Erciyes University, Kayseri, Turkey

The corpus callosum is the main neural pathway that connects the two cerebral hemispheres. The anatomy and embryology of this commissural structure have been studied extensively. It develops between the 8th and 20th week of gestation. Basic knowledge of normal development is necessary to the under- standing of carpus callosum abnormalities. If the normal developmental process is disturbed, the corpus callosum may be completely or partially absent. Agenesis of corpus callosum is usually associated with other anomalies such as congenital brain anomalies, chromosomal abnormalities, and metabolic diseases. In this study, we encountered partial agenesis of corpus callosum using magnetic resonance (MR) imaging. Analysis of corpus callosum provides important information about the embryogenesis of brain anomalies and may help in distinguishing between in utero and perinatal brain development.

Keywords: Corpus callosum, partial agenesis, brain, magnetic resonance

\section{P-48}

\section{Using a Wordpress blog as a platform for anatomy education- Yeditepe experience}

Yücel K

Department of Anatomy, Faculty of Medicine, Yeditepe University, istanbul, Turkey

The experience of Department of Anatomy of Medical School of Yeditepe University on using Wordpress blogs as platforms for anatomy education for students of medicine, dentistry and pharmacy in the school year 2011-2012 is presented. A separate blog at www.wordpress.com was created for each anatomy class; first and second year medical students, first year pharmacy students, first and second year dentistry students. The lectures were uploaded as powerpoint presentations and Word documents as a post in the blog, at least one day prior to the lecture. A dynamic blog has several advantages over using a static webpage to upload lectures. These advantages include, but not restricted to, easy way of tracking the number of downloads prior to the lecture, being able to moderate the comments, adding different features to the post related to the lecture e.g., YouTube videos, relevant TUS (Medical Speciality Exam) questions, the survey feature of the blog, having a separate link for each lecture, etc. Blogging has recently been used in medical practice for a variety of reasons. This trendy platform of social media can also be used for anatomy education in order to contribute to interactive learning with a comphrensive approach by getting benefits from other social media channels such as YouTube, online anatomy atlases. Further studies are needed to improve the use of blogging in medical education.

Keywords: Anatomy education

\section{P-49}

Anomalous fusion between the lunate and triquetrum: a case presentation

Öğüt $E^{*}$, Ürgüden $M^{* *}$, Oruç $F^{* * *}$, Oğuz $N^{*}$, Yıldırım FB*

*Department of Anatomy, Faculty of Medicine, Akdeniz University, Antalya, Turkey; **Department of Orthopedy and Traumatology, Faculty of Medicine, Akdeniz University, Antalya, Turkey; ***Department of Orthopedy and Traumatology, Medikal Park Hospital Complex 
Various synostoses between carpal bones reported. The most common carpal coalition is between carpal lunate and triquetrum called as os lunatotriquetrum. The incidence of these anomalies in general population is between $\% 0.08$ and $\% 0.13$. Lunato-triquetral coalitions are nearly always asymptomatic and six types of lunato-triquetral fusion are described. This bone anomaly is mostly bilateral but more common on the left side when unilaterally present. A Case thirty-seven year old man who complaints avultion fracture of the lateral and distal part of the right radius so performed CT imaging result showed a lineer dancity of internal fixator from medial side of the wrist between the triquetrum and lunate. An internal fixator reported between the lunato-capitatum and lunato-radius and also lunato-scaphoideum. But pieced fracture was noted in scaphoideum. There is no trace of any pathological process and no other abnormality exists in the skeleton of hands. Clinicians should keep in mind the existence of lunato-triquetral coalitions which has several types and nearly always asymptomatic.

Keywords: Carpus, carpal fusion, lunato-triquetrum.

\section{P-50}

\section{Berretini anastomoses in fetuses}

Kara A, Elvan Ö, Öztürk NC, Öztürk H

Department of Anatomy, Faculty of Medicine, Mersin University, Mersin, Turkey

The communication(s) between common digital nerves that arise from the ulnar and median nerves in the palmar surface of hand is called 'ramus communicans cum nervi ulnari' (also called Berretini anastomoses, (BA) or reversed martin gruber anastomoses). In the literature, the Berrettini anastomoses have been described as a normal finding, or an anatomical variation, or an anomalous branch, or they have been totally ignored. These anastomoses have been recently revisited and previous descriptions have been unified in adult cadavers. Differently, the present study was conducted to examine the incidence and patterns of BA in human fetuses. Palmar surfaces of hands of 40 fetuses (23 female, 15 male, and 2 unknown) were dissected bilaterally for this purpose. Berretini anastomoses were observed in 17 fetuses (42.5\%), (unilaterally in 12 (30\%), and bilaterally in $5(12.5 \%)$ ). When all sides (80 sides) were taken into account, the side incidence was found as $27.5 \%$. The general pattern of these anastomoses was observed as diverging from the branches of the ulnar nerve innervating the 4th and 5 th digits and uniting with the branches of the median nerve innervating the $3 \mathrm{rd}$ and 4th digits. Knowledge of these anastomoses is crucial for hand surgeons and neurologists.

Keywords: Median nerve, ulnar nerve, fetus, Berretini anastomoses

\section{P-51}

Martin-Gruber anastomoses and its relation with accessory flexor forearm muscles in fetuses

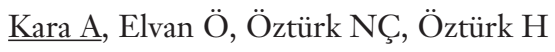

Department of Anatomy, Faculty of Medicine, Mersin University, Mersin, Turkey
Martin-Gruber anastomoses (MGA) is a neural connection between the median and ulnar nerves in the forearm that provide variations in the innervation of the intrinsic hand muscles. These anastomoses were reported to convey motor axon from the median nerve to the unlar nerves in literature. The present study was conducted to examine the incidence and morphology of MGA in human fetuses. Anterior aspect of the forearms of 40 fetuses ( 23 female, 15 male, and 2 were unknown) were dissected bilaterally for this purpose. The MGAs were observed in 17 fetuses (42.4\%), (unilaterally in 11 (27.5\%), and bilaterally in $6(15 \%))$. When all sides (80 sides) were taken into account, the side incidence was found as $28.8 \%$ ( 23 sides). We also observed that the accessory head of flexor pollicis longus (in 5 forearms) and accessory head of flexor digitorum profundus muscle (in 6 forearms) was crossing the MGA anteriorly. At one side there was coexistence of both of these accessory muscles and MGA. These muscles are thought to be potential compressors of MGA. The MGA has clinical significance for understanding median nerve lesion and the carpal tunnel syndrome. Therefore, knowledge of these anastomoses is crucial for clinicians.

Keywords: Median nerve, ulnar nerve, fetus, Martin-Gruber anastomoses, accessory heads of flexor forearm muscles

\section{P-52}

\section{Incidence of the accessory head of flexor digitorum profundus muscle and its usage in hand surgery}

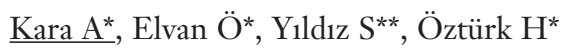

*Department of Anatomy, Faculty of Medicine, Mersin University, Mersin, Turkey; **Department of Anatomy, Gülhane Military Medical Academy, Ankara, Turkey

The present study was conducted to ascertain the incidence of the accessory head of flexor digitorum profundus muscle (ahFDP), to examine its morphometric properties and to evaluate its usage in hand surgery. We carried out this study in Mersin University Medical Faculty and Gulhane Military Medical Academy, in both fetuses and adult cadavers. Ninety forearms of 45 fetuses and 48 forearms of 22 adult cadavers plus 4 amputated cadaver upper limbs were dissected for this purpose. The ahFDP was observed in $14 \%$ of the fetal and $30.8 \%$ of adult cadaver forearms. It was found in 3 fetal and 5 adult cadavers bilaterally, and in 7 fetal and 6 adult cadavers unilaterally and in 2 amputated upper limbs. Tendon measurements were carried out only in the adult cadaver forearms. The average total length (muscle belly plus tendon) of ahFDP and its tendon length were measured as $135.74 \pm 54.94$ and $62.27 \pm 44.94 \mathrm{~mm}$ respectively. And the average tendon width was measured as $3.45 \pm 1.0 \mathrm{~mm}$. According to its morphometric properties, sufficiency of the tendon of ahFDP is evaluated for tendon transfer and repair in hand surgery.

Keywords: Accessory head of flexor digitorum profundus muscle, fetus, cadaver, tendon transfer, hand surgery 
S142 14th National Congress of Anatomy, 28 June-1 July 2012, Ankara, Turkey

\section{P-53}

Immunohistochemical investigation of the effect of green tea and vitamin $\mathrm{E}$ to the rat blood-brain barrier and to the inflamation in streptozotocin induced diabetes mellitus

Bahçelioğlu $M^{*}$, Take $\mathrm{G}^{* *}$, Gözil R* $\mathrm{R}^{*}$, Buru E*, Helvacioğlu $\mathrm{F}^{* * *}$, Çalgüner $\mathrm{E}^{*}$, Erdoğan $\mathrm{D}^{* *}$

*Department of Anatomy, Faculty of Medicine, Gazi University, Ankara, Turkey; **Department of Histology and Embryology, Faculty of Medicine, Gazi University, Ankara, Turkey; ***Department of Histology and Embryology, Faculty of Medicine, Başkent University, Ankara, Turkey

Diabetes mellitus (DM) is a chronic metabolic disease. DM complications have been linked to oxidant stress, decreased consumption of oxygene in mitochondrions and antioxidant defense of the body. Our aim was to investigate the possible effect of the antioxidant theraphy such as green tea and vitamin $\mathrm{E}$ in frontal cortex the glial fibrillary acidic protein (GFAP) activity to evaluate the distribution of astrocytes with are effective in blood-brain barrier and nuclear factor $\kappa \beta(\mathrm{NF}-\kappa \beta)$ activity wich is known to increase in inflamation related to diabetes. In this study, 54 Wistar albino rats, divided into 9 groups. After 6 weeks of the streptozotocin (STZ) injection, and then 4 weeks group 7, 8 and 9 received orally green tea $(300 \mathrm{mg} / \mathrm{kg})$ and vitamin $\mathrm{E}(0.4 \mathrm{mg} / \mathrm{kg})$. Group 1:Normal control, Group 2: Na sitrat, Group 3: Green tea, Group 4: Vitamin E, Group 5: Green tea + vitamin E, Group 6: Diabetic control (STZ (50 mg/kg), single injection), Group 7: STZ + green tea, Group 8: STZ + Vitamin E, Group 9: STZ + green tea + vitamin E. At the end of the $10 \mathrm{week}$, all the animals were sacrified. Sections for immunohistochemical examination of anti-GFAP and anti-NF- $\kappa \beta$ to were done. Slides were examined with Photo-light microscope. In diabetic control group, GFAP immunoreactivity was observed to be interrupted in pia-glial membrane and around vessels. Administration of antioxidant agents was not sufficiently effective for the restructuring of the blood-brain barrier. In diabetic control group, NFк $\beta$ immunoreactivity was very prominent in neurons and glial cells and antioxidant therapy decreased the immunoreactivity of $\mathrm{NF} \kappa \beta$ in these structures. We observed an interrupted immunoreactivity of GFAP in pia-glial membrane and around the vessels of brain frontal cortex while NFK $\beta$ staining was increased in neurons and glial cells related to DM. We determined that the antioxidant theraphy was not effectively decreasing these effects seen in diabetic group.

Keywords: Diabetes mellitus

\section{P-54}

Immunohistochemical investigation of the effect of oral methylphenidate administration in rat cerebellum dopamine 2 receptor activity three month after the cessation of the drug

Bahçelioğlu $M^{*}$, Take G**, Gözil R*, Buru E*, Helvacioğlu $\mathrm{F}^{* * *}$, Saraç GN**, Çalgüner $\mathrm{E}^{*}$, Erdoğan $\mathrm{D}^{* *}$

*Department of Anatomy, Faculty of Medicine, Gazi University, Ankara, Turkey; **Department of Histology and Embryology, Faculty of Medicine, Gazi University, Ankara, Turkey; ***Department of Histology and Embryology, Faculty of Medicine, Başkent University, Ankara, Turkey
Ritalin (methylphenidate) is widely used in treatment of children with ADHD. Our aim was to determine effect of methylphenidate in rat cerebellum after several months following termination of drug administration. In experiment, $110 \mathrm{~g}$ $( \pm 20), 36$ female Wistar albino rats divided into two different dose (10-A1, 20-A2 mg/kg) and time group (three months drug administration -B1- and three months follow-up after drug cessation -B2-) with control groups (A0,B0), were used. Pre-pubertal (35 days old) rats were treated orally with $\mathrm{MPH}$ dissolved in saline solution for 5 days/week during 3 months. At the end of third month, A1, A2 group rats were perfused and cerebellum was removed. B1, B2 group were feeded for 3 more months without any substance administration and they followed same procedure. For immunohistochemical studies, cerebellar tissues marked with anti-dopamine-2R primary antibody. Sections were evaluated by Photo-light microscope (DM4000B IAS, Leica,Germany). In A0 group, dopamine-2R immunoreactivity was observed in scarce purkinje cells and few neurons of molecular and granular layers. In A1 group, most purkinje cells displayed moderate dopamine-2R immunoreactivity while few cells showed negative staining. Number of immunostained cell was increased in granular layer. In A2 group, strong widespread staining was observed overall cerebellum. In BO group, immunoreactivity was similar to A0 group in purkinje cells but widespread immunostaining in granular layer. In B1 group, immunoreactivity in purkinje cells displayed resemblance to A1 group in molecular and granular layer neurons; immunostaining was similar to B0 group. In B2 group, immunostaining was decreased. Immunostaining in molecular layer neurons of this group was similar to B0 group but the staining was strong in granular layer neurons. We observed that the recovery of structural findings in cerebellum were dose-related. In normal dose treated group, dopamine-2R staining was reversed totally but especially in high dose treated group, recovery wasn't possible in granular layer neurons

Keywords: Methylphenidate, cerebellum, immunohistochemistry

\section{P-55}

The angle of torsion of the humerus head to determine by MRI method

Tellioğlu MA, Karakaş S, Taşkın F

*Department of Anatomy, Faculty of Medicine, Adnan Menderes University, Aydin, Turkey; ${ }^{*}$ Department of Histology and Embryology, Faculty of Medicine, Adnan Menderes University, Aydın, Turkey

The torsion angle values of the calculation method of MRI in healthy volunteers and aimed to the detection of gender-related differences. MRI method for the calculation of the angle of torsion of the humerus, 36 healthy adults (18 men, 18 women between the ages of 20-68) were included in the study. HTA axial MRI images were calculated. Obtained data were analyzed with SPSS (14.0 version) program. Compliance with all of the normal distribution of data Kolmogorov-Smirnov test was applied. MRI results, the differences between sexes and age 
groups, students t test. 36 healthy adults (18 men between the ages of $20-68,18$ females) to be made, calculated by the method of MRI, HTA, women mean $18.1 \pm 12.6\left(-4^{\circ}\right.$ to $\left.36^{\circ}\right)$, mean $19.6 \pm 12.7$ for males $\left(2^{\circ}\right.$ to $\left.41^{\circ}\right)$, respectively. In all healthy volunteers, HTA 'average value was $18.9^{\circ} \pm 12.5$ ( -4 to $41^{\circ}$ ) respectively. The average torsion angle of $21.9^{\circ} \pm 12.9$ individuals between the ages of 20-40, the average torsion angle of $15.8^{\circ} \pm 11.6$ individuals over the age of 40 , respectively. According to the evaluation of the results Student's t-test, no significant differences were found between genders and age groups. The results obtained from healty volunteers, the angle be measured MRI showed that the method successfully. Prosthetic replacement of shoulder joint prosthesis design and in terms of our results could be used as a reference. In addition, recurrent anterior shoulder joint dislocation syndrome related pathologies such as chronic shoulder pain in patients; we think that may be useful in assessing upper extremity athletes.

Keywords: Humerus, torsion angle, $M R G$

\section{P-56}

Relations of morphometric measurements of hand and sex and stature

Çetince $S^{*}$, Karagöz ÖC $C^{*}$ Kılıç A*, Pekşen B*, Uysal FR*, Öztuna $\mathrm{D}^{* *}$, Açar Hi்***

*Medical Student, Faculty of Medicine, Ankara University Ankara, Turkey; **Department of Biostatistics, Faculty of Medicine, Ankara University, Ankara, Turkey; $* * *$ Department of Anatomy, Faculty of Medicine, Ankara University, Ankara, Turkey

The most important problem is identification of a person or dead body for forensic science experts. Stature and sexuality are some of the most important parameters for identification. We can obtain valuable informations about length and sexuality of person, using morphometrics measurements of hand. The aim of the study to present relations of morphometry of the hand and sexuality together with length also contribute to the small number of studies in the literature. This study is a cross sectional study. The work was conducted on 277 students from among 1-2-3 of the period in the Ankara University Medical School, using random sampling. Demographic characteristics (sexuality, length and age) have been collected using face to face interview method. Morphometric measurements of the hand have been measured with digital caliper. For the assessments; Student's T test, Chi- square test and One-Tailed Variance were analyzed, using SPSS 11.5 program in this study. Students who attend to research median age is 20 (minimum 18, maximum 27); and \%51.3 of them are women. There is a connection between right and left hand length and person's height which makes sense as statistical $(r=0.781$ and $r=0.816$; $\mathrm{p}<0.001$ ). Right and left hand length, width hand length/ height rate and hand width/ height rate means are higher for male $(\mathrm{p}<0.01)$. There is a difference between right and left hand for hand width and palmar index $(\mathrm{p}<0.01)$. 2nd finger/4th finger length rate and 3rd finger/5 th finger length rate are the most sensitive conclusion for sex difference. Morphometric feature of hand can be counted as characteristic factor for identification of person.

Keywords: Hand morphometry, hand index, palmar index, forensic medicine, identification

\section{P-57}

The investigation of the relationship between finger length, abduction angle and hand span length

Kosif $\mathrm{R}^{*}$, Bulut $\mathrm{E}^{* *}$, Kavvasoğlu $\mathrm{C}^{* *}$, Yılmaz F**

*Faculty of Medicine, Abant lzzet Baysal University, Bolu, Turkey; ${ }^{*}$ Medical Student, Faculty of Medicine, Abant Izzet Baysal University Bolu, Turkey

Abduction angles on hands, hand span and finger lengths are important for specific occupations. The correlations between finger lengths, abduction angles, and hand span lengths are investigated in this study. 34 right handed male students in Medical Faculty participated in this study. Five finger lengths, four abduction angles according to middle line and hand span length are measured on the right hand radiographs taken in the maximum abduction position and the values are statistically evaluated in SPSS 13.0 programme. Evaluating finger lengths and abduction angles, there was only a negative correlation between thumb length and abduction angle of little finger $(r=-$ 429). All of the finger lengths were positively correlated with the length of hand span. Also there was positive correlation between thumb abduction angle and hand span and between ring finger abduction angle and little finger abduction angle (respectively $r=0,649, r=0,382$ ). Knowledge about the correlation between finger lengths, abduction angles and hand span length will be useful for employees who use computer, play a musical instrument, anthropology and work in biomechanics of hand. This study shows that there is no positive correlation between finger length and abduction angle, also thumb abduction angle enlarges the length of hand span.

Keywords: Finger length, abduction angle, hand span, relationship

\section{P-58}

\section{Behavioral lateralization and digit ratio: a preliminary assessment}

Ertuğrul Özener B*, Otağ I**, Koşar Mï***

*Institute of Social Sciences, Cumhuriyet University, Sivas, Turkey; **Vocational School of Health Services, Cumhuriyet University, Sivas, Turkey; ***Department of Anatomy, Faculty of Medicine, Cumhuriyet University, Sivas, Turkey

It is believed that testosterone has a role in the development of the fourth finger, while estrogen in the development of the second finger in the prenatal period. Thus, concentration of high testosterone and low estrogen $(\mathrm{T}>\mathrm{E})$ reduces the ratio of the second finger to the fourth finger, while high estrogen concentration $(\mathrm{T}<\mathrm{E})$ increases this ratio. It is claimed, moreover, that high 
S144 14th National Congress of Anatomy, 28 June-1 July 2012, Ankara, Turkey

testosterone level increases the rate of the fetus being left-handed. The aim of this study is to determine whether there is a relationship between digit ratio and right-handedness and left-handedness. The study measured the length of the second and fourth finger of 15 left-handed young individuals ( 7 males, 8 females) and 15 right-handed young individuals ( 7 males, 8 females) with $0.01 \mathrm{~mm}$ accuracy and calculated the ratio of the second finger to the fourth (2D:4D). The study found that the digit ratios of the individuals in both groups differed significantly according to sex, while this difference was not significant between the righthanded and left-handed individuals. While prenatal steroid levels showed a significant difference in the digit ratio (2D:4D) between sexes, no significant relationship was found between digit ratio and behavioral laterality in neither of the sexes. No significant effect of prenatal steroid levels on behavioral laterality was observed. However, it could be stated that examining this relationship in larger groups might reveal better results.

Keywords: Digit ratio, 2D:4D, left-handedness, prenatal steroids

\section{P-59}

Oxidative tissue damage in the prefrontal cortex of pinealectomized rats and protective effects of melatonin hormone

Kuş MA* Çolakoğlu $\mathrm{S}^{* *}$, Karaca Ö̈ ${ }^{* * *}$, Gülcen B*** Kuș I*** , Sarsilmaz $M^{* * * *}$

*College of Health, Mehmet Akif Ersoy University, Burdur, Turkey; **Department of Anatomy, Faculty of Medicine, Düzce University, Düzce, Turkey; $* * *$ Department of Anatomy, Faculty of Medicine, Balıkesir University, Balıkesir, Turkey; $* * * *$ Department of Anatomy, Faculty of Medicine, Şifa University, Izmir, Turkey

An attempt was made to investigate the tissue samples of prefrontal cortex from pinealectomized rats and melatonin applied rats following pinealectomy in biochemical basis. Twenty-one, male Wistar rats were divided equally into three groups. Rats in Group I were used as control (sham-pinealectomy). Rats in Group II were pinealectomized. Rats in Group III were injected daily with melatonin $(1 \mathrm{mg} / \mathrm{kg})$ by intraperitoneally for 3 months following pinealectomy. At the end of experimental period, all rats were killed by decapitation and the prefrontal cortexes of rats were removed. Superoxide dismutase (SOD), glutathione peroxidase (GSH-Px) enzyme activities and malondialdehyde (MDA) levels were determined at biochemical levels in the prefrontal cortex specimens. The levels of SOD and GSH-Px in the prefrontal cortex tissue were significantly decreased and MDA levels were significantly increased in pinealectomized rats compared with those of the controls $(\mathrm{p}<0.05)$. However, increased activities of SOD and GSH-Px enzymes and decreased levels of MDA were detected in the rats administered melatonin following pinealectomy $(\mathrm{p}<0.05)$. Pinealectomy-induced oxidative damage in the prefrontal cortex tissue in rats was prevented by administration of melatonin hormone.

Keywords: Prefrontal cortex, melatonin, pinealectomy

\section{P-60}

Stereological assessment of the protective effect of atropine on the purkinje cells of the cerebellum damaged by fenthion

Baş $\mathrm{O}^{*}$, Aslan $\mathrm{A}^{* *}$, Söztanacı US*, Sönmez ÖF***,

Tümkaya $L^{* * * *}$, Yağmurca $M^{* * * * *}$, Toktaş $M^{* * * * * *}$

*Department of Anatomy, Faculty of Medicine, Rize University, Rize, Turkey; **Department of Physiology, Faculty of Medicine, Rize University, Rize, Turkey; ***Department of Neurosurgery, Samsun Training and Research Hospital, Samsun, Turkey; $* * * *$ Department of Histology and Embryology, Faculty of Medicine, Rize University, Rize, Turkey; $* * * * *$ Department of Histology and Embryology, Faculty of Medicine, Fatih University, Ankara, Turkey; $* * * * * *$ Department of Anatomy, Faculty of Medicine, Fatih University, Ankara, Turkey

Organophosphates are drugs used as pesticides in agriculture. Physicians usually encounter patients with organophosphate intoxication because of accidental exposure to high doses of the drug or suicide attempt. Because of their high toxicity, organophosphates cause severe morbidity and mortality. The aim of our study was to investigate the toxic effect of fenthion on purkinje cells in cerebellum by using stereologic methods and to determine whether atropine has protective effects. Eighteen female adult Wistar albino rats were divided into three groups. Control group received a single dose of $0.8 \mathrm{~g} / \mathrm{kg}$ subcutaneous saline followed $2 \mathrm{mg} / \mathrm{kg} / \mathrm{h}$ saline intraperitoneally for four hours. Fenthion group received single dose of 0.8 $\mathrm{g} / \mathrm{kg}$ subcutaneous fenthion followed by $2 \mathrm{mg} / \mathrm{kg} / \mathrm{h}$ saline intraperitoneally for four hours. Fenthion+atropine group received a single dose of $0.8 \mathrm{~g} / \mathrm{kg}$ subcutaneous fenthion followed by $2 \mathrm{mg} / \mathrm{kg} / \mathrm{h}$ atropine intraperitoneally for four hours. On the fifth day, animals were sacrificed and sequential sections of cerebellum were stained with Cresyl Fast Violet. Optical fractionator sampling method was used to estimate total neuronal number. The results showed that purkinje cell number in fenthion group rats were slower than control and fenthion+atropine groups. Compared to fenthion+atropine group, a statistically significant decrease was observed on neuron number in fenthion group. There were no statistically significant differences between in control and fenthion+atropine groups' neuron number of purkinje cell in cerebellum. Fenthion significantly reduces total purkinje neuronal number in cerebellum, increases apoptosis, Toxic effects of fenthion can be reduced completely or partly by atropine.

Keywords: Fenthion, cerebellum, stereology, atropine

\section{P-61}

Histological changes in livers of pregnant rats and offspring of epileptic lamotrigine and folic acid effects on the liver of newborn

$\underline{\text { Soysal } H^{*}}$, Eşrefoğlu $M^{* *}$, Ekinci $\mathrm{N}^{* * *}$

*Department of Anatomy, Faculty of Medicine, Adıyaman University, Adıyaman, Turkey; Bezmialem Foundation University, **Department of Embryology and Histology, Faculty of Medicine, istanbul, Turkey; ***Department of Anatomy, Faculty of Medicine, Inönü University, Malatya, Turkey 
Determine of effects of lamotrigine (LTG) and folic acid (FA) on changes of liver tissue in offspring of epileptic pregnant rats. 16 Wistar-Albino pregnant rats were used in the study. intraperitoneally Lamotrigine $2 \mathrm{mg} / \mathrm{kg}$ and orally $400 \mu \mathrm{g} / \mathrm{kg}$ folic acid were administrated to pregnant rats at each day of pregnancy period. Acute epileptic seizures was produced by experimental epilepsy model at the 13 th day of pregnancy. We evaluated by light microscope. of liver tissue in offspring rats at 0th, 21th, and 38 th days after birth. The studies on the light microscobe showed that the new born liver lobules are not properly matured; central vens and the sinusoids are usually large and irregular in the control group.In the very few portal areas a tiny bile duct, artery and ven were observed. Typically, the hepatocytes patterned in the group of cells vacuolization was remarkable. No change observed on the newborns of the rats had the epileptic attach comparing to the control groups newborns. In the experimental groups, there was no evidence of any different from the offspring rats at 0 th, 21 th, and 38 th days after birth. It has been observed that all the studies under the light microscope on the epileptic attack during the pregnancy and consuming LTG ve FA during pregnancy caused no pathologic demage and no maturing change on the new borns' livers.

Keywords: Epilepsy, folic acid, liver, lamotrigine, newborn

\section{P-62}

The histological and biochemical effects of lamotrigine, phenytoin and folic acid on the pregranat rats having epileptic attack

Soysal H*, Doğan $Z^{* *}$, Eşrefoğlu $M^{* * *}$, Türkoz $Y^{* * * *}$

*Department of Anatomy, Faculty of Medicine, Adiyaman University, Adiyaman, Turkey; ${ }^{*}$ Department of Anatomy, Faculty of Medicine, Inönü University, Malatya, Turkey; ***Department of Histology Embriyology, Faculty of Medicine, Bezmialem Vakıf University, Istanbul, Turkey; $* * * *$ Department of Biochemistry, Faculty of Medicine, Inönü University, Malatya

It is aimed to observe the effects of the LTG and PHT on the fetal hepar tissues of the pregnant rats that the experimental epilepsi is practised on. 27 rats have been selected and grouped into 10 to be observed in the study. Each day of the pregnancy $2 \mathrm{mg} / \mathrm{kg} \mathrm{LTG}, 25 \mathrm{mg} / \mathrm{kg}$ PHT and $400 \mu \mathrm{g} / \mathrm{kg} \mathrm{FA}$ are dispensed and applied. On the 13th day of the pregnancy, experimental epilepsi is implemented. Following the birth of 210 new born rats, hepar tissues' antioxidant zymase activity, malondialdehid and redukte glutatyon (GSH) levels are measured. The results are evaluated on the level of light microscope. The observations regarding the oxsidative demage, the GSH level is decreased and the MDA level is increased in the PHT group comparing to the control group. The application of Folic asid helped with increasing the GSD level and decreasing the MDA level. In the LTG group the GSH and the MDA levels are increased comparing to the control level. Application of the LTG amongst the experimental apilepsia group helped with increasing the GSH levels which had decreased before. The studies on the light microscobe showed that the new born liver lobules are not properly matured; central vens and the sinusoids are usually large and irregular in the control group.In the very few portal areas tiny bile duct, artery and ven were observed. Typically, the hepatocytes patterned in the group of cells vacuolization were remarkable. No change observed on the newborns of the rats had the epileptic attach comparing to the control groups newborns. In the PHT group some sinuzoidal engorgement has been observed in addition to the rest of the observations. It has been observed that the epileptic attack and the consuming PHT during the pregnancy period are causing oxidative demage in the fetal hepar whereas LTG and folic acid are acting as a preservative on the demage. All the studies under the light microscope on the epileptic attack during the pregnancy and consuming PHT, LTG ve FA during pregnancy caused no pathologic demage and no maturing change on the new borns' livers.

Keywords: Antioxidant, lamotrigine, phenytoin and folic acid

\section{P-63}

Unilateral variation of sural nerve: a cadaveric study

Kaçar D, Barut Ç

Department of Anatomy, Faculty of Medicine, Bülent Ecevit University, Zonguldak, Turkey

The sural nerve is formed by uniting of lateral sural cutaneous nerve and medial sural cutaneous nerve in mid-section of the posterior surface of leg, extending along with lesser saphenous vein until the posterior aspect of outer malleolus. A unilateral variation of sural nerve was encountered. We identified a superiorly located sural nerve formation $2-3 \mathrm{~cm}$ under the popliteal fossa during the dissection of right posterior surface in a white adult female cadaver. A digital caliper with a resolution of 0.01 $\mathrm{mm}$ was used for measurements. The length of lateral sural cutaneous nerve originating from common fibular nerve was $11.76 \mathrm{~mm}$ and medial sural cutaneous nerve branching from tibial nerve was $13.88 \mathrm{~mm}$. We identified that medial sural cutaneous nerve, normally branching from between the two heads of gastrocnemius muscle, branches at a superior region, in the lower margin of popliteal fossa. This is a unilateral variation in which sural nerve forms superior to its usual site of formation. Clinically, these types of variations are vital in diagnostic invasive interventions, angiographic applications and treatment surgeries of the region. The recognition of these variations is important in minimizing the surgical complication in surgeries of the region.

Keywords: Sural nerve, variation, cadaver, unilateral

\section{P-64}

The achilles hells in melatonin: astma

Minareci E*, Kaya $\mathrm{Y}^{* *}$, Yıldırım FB**

*Department of Pharmacology and Toxicology, Faculty of Medicine, Akdeniz University, Antalya, Turkey; Department of Anatomy, Faculty of Medicine, Akdeniz University, Antalya, Turkey 
Asthma is a clinical syndrome characterized by chronic airway inflammation, airway responsiveness, and expiratory airflow limitation. Nocturnal symptoms and decreases in lung function are common aspects of the asthma clinical syndrome. Nocturnal symptoms also appear to be associated with asthma-related mortality. In addition to its importance to the regulation of human circadian rhythms, an accumulating body of evidence also suggests that melatonin is also involved in the regulation of smooth muscle tone. For this reason, this study aimed to evaluate contraction and relaxation responses in tracheal smooth muscle rings obtained from rats treated with melatonin. Following administration of melatonin $(50 \mathrm{mg} / \mathrm{kg} / \mathrm{day})$ at the same time every day for 6 weeks, in vitro organ bath experiments were performed with rat tracheal preparations exposed to contractile (acetycholine and serotonin) and relaxant (theophylline and papaverine) agents. Melatonin treatment strengthened contraction responses, but did not affect relaxation responses in rat tracheal preparations. We think that melatonin might play a role in the pathogenesis of nocturnal asthma. Therefore, clinicians should be aware of the importance of melatonin to nocturnal exacerbation of asthma symptoms and alert asthmatic patients that use exogenous melatonin supplementation of its potential negative effects.

Keywords: Melatonin, bronchial asthma, circadian rhythm, trachea

\section{P-65}

\section{Location of internal acustic canal in relation to} anatomical landmarks

İçke $C^{*}$, Özer $E^{* *}$, İçke $S^{* * *}$

*Department of Anatomy, Faculty of Medicine, Dokuz Eylül University, Izmir, Turkey; **Department of Neurosurgery, Faculty of Medicine, Dokuz Eylül University, Izmir, Turkey; ***Department of Neurosurgery, Türkan Özilhan Emergency Care and Traumatology Hospital, Izmir, Turkey

Location of internal auditory canal (IAC) is of importance during middle cranial fossa surgery. It's important to know its location in relation to other anatomical landmarks. We used 24 cranial bases (48 specimens), 38 right-side and 33 left-side temporal bones; 119 specimens overall. Medial (MUMPB) and lateral (LUMPB) edges of upper margin of petrous of bone (UMPB) were determined. Medial (ME), lateral (LE) and upper medial (UME) points of entrance (E) of IAC were identified. UMPB, ME - MUMPB, LE - LUMPB, UME - UMPB distances were measured. UMPB was divided into three equal parts and position of $\mathrm{E}$ was evaluated in relation to these parts. The length of UMPB were measured as 56,29 $\pm 4,37 \mathrm{~mm}$; ME - MUMPB and LE - LUMPB UME - UMPB distances were $15.61 \pm 2.69 \mathrm{~mm}$ and $34.37 \pm 3.71 \mathrm{~mm}$ respectively. UME - UMPB distance was measured as $4.87 \pm 2.19 \mathrm{~mm}$. It was observed that $\mathrm{O}$ was located in both medial and middle parts in $95 \%$, in middle in $4.2 \%$ and in medial in $0.8 \%$ of cases. Results of this study will help to determine of location of IAC during temporal bone surgery.

Keywords: Middle cranial fossa, internal auditory canal, temporal bone

\section{P-66}

Angular description of the localization of the internal acustic canal

İçe $C^{*}$, Özer E** Çırpan $S^{*}$

*Department of Anatomy, Faculty of Medicine, Dokuz Eylül University, izmir, Turkey; **Department of Neurosurgery, Dokuz Eylül University, izmir, Turkey

Internal auditory canal (IAC), is a canal of $1.5 \mathrm{~cm}$ length in the petrous part of the temporal bone. Exact knowledge of the localization of IAC is important in the surgical interventions. We used 24 cranial bases (48 specimens), 38 right-side and 33 left-side temporal bones; 119 specimens overall. The lengths of the lateral, and medial walls and the entrance pole of the IAC were measured. Taking into consideration the localization and structural properties of IAC, it was described as a triangle. Knowing the three sides of this triangle, using the law of cosines, the angles of it were calculated. A angle: The angle between the M-O; B angle: The angle between the L-O; $\mathrm{C}$ angle: The angle between the $\mathrm{M}-$ L. The L, M, O lengths were measured as $10.03 \pm 1.65 \mathrm{~mm}$, $13.43 \pm 1.78 \mathrm{~mm}, 8.30 \pm 1.38 \mathrm{~mm}$ in order. The angles of the triangle were calculated as $\mathrm{A}=47.54 \pm 11.09^{\circ}, \mathrm{B}=95.01 \pm 15.64^{\circ}, \mathrm{C}=$ $37.43 \pm 8.86^{\circ}$. Localization of the IAC is key to the middle cranial fossa (MCF) approach. The results of this study will guide to define of the location of the IAC in the temporal bone.

Keywords: Internal auditory canal, angle, temporal bone

\section{P-67}

\section{Radiographic findings of lumbar disc degeneration}

$\underline{\text { Karabulut } \text { Ö}^{*}}$, Hatipoğlu ES*, Karabulut $Z^{* *}$, Tuncer $\mathrm{MC}^{*}$, Açıkgöz A***

*Department of Anatomy, Faculty of Medicine, Dicle University, Diyarbakır, Turkey; ${ }^{* *}$ Depatment of Physiatry, Özel Veni Vidi Hospital, Diyarbakır, Turkey; ***Depatment of Radiology, Özel Veni Vidi Hospital, Diyarbakır, Turkey

Radiography is a widely accepted and low cost method to measure structural changes of bone. Lumbar disc degeneration is characterized by the presence of osteophytes, end plate sclerosis and disc space narrowing in lumbosacral radiographic images. The aim of our study was to assess the characteristic radiologic findings of lumbar disc degeneration. Our study was designed to assess lumbar osteophytes, disc space narrowing and end plate sclerosis. The study group included one thousand male patients. Anterioposterior-lateral spinal radiography of each vertebral level from L1-L2 to L5-S1 was assessed. The presence and grades of osteophytes, disc space narrowing and end plate sclerosis were evaluated by the observers. Pearson correlation test was used for the statistical analysis. In lumbosacral radiographs $90.6 \%$ of all lumbar vertebral levels showed osteophytes, $87.5 \%$ end plate sclerosis and $68.2 \%$ disc space narrowing. There was a significant relationship among the radiographic features by Pearson correlation test. In addition the results of this study showed that pairs of osteophytes frequently formed in the upper 
lumbar vertebrae (L1-L2, L2-L3). Our study demonstrates that the measures using software analysis of vertebral radiographic images compare the lumbar disc degeneration findings and show a statistically significant correlation.

Keywords: Lumbar disc degeneration, osteophytes, digital radiography

\section{P-68 \\ Effects of short, moderate and long term swimming exercise on extra-seizure behavioral properties of WAG/Rij rats with absence epilepsy}

Çakıl D*, İnanır $\mathrm{S}^{* *}$, İnanır $\mathrm{A}^{* * *}$, Aygün $\mathrm{H}^{*}$, Baykan $\mathrm{H}^{* * * *}$

*Department of Physiology, Faculty of Medicine, Gaziosmanpaşa Universty, Tokat, Turkey; **Department of Psychiatry, Faculty of Medicine, Gaziosmanpaşa Universty, Tokat, Turkey; ***Department of Physical Medicine and Rehabilitation, Faculty of Medicine, Gaziosmanpaşa Universty, Tokat, Turkey; ****Psychiatry Clinic, Ümraniye Education and Research Hospital, Istanbul, Turkey

Wag/Rij rats with absence epilepsy are reported to have different behavioral properties compared to Wistars. The aim of this study was to evaluate effects of short, moderate and long term swimming exercise on locomotor activity and anxiety states of WAG/Rij rats $6 \mathrm{Wistar}$ and $24 \mathrm{WAG} / \mathrm{Rij}$ male rats were used throughout the study. WAG/Rij rats were divided into 4; as 1 control and 3 experimental groups. Experimental groups were submitted to daily swimming exercise for 90 days, 15, 30 and 60 minutes/day; respectively. Locomotor activity and anxiety states of all groups were evaluated with open field test. WAG/Rij rats were found to have significantly high locomotor activity compared to Wistars, which was evaluated by counting the number of squares crossed by the animal. But there was no significant difference between anxiety states of two strains, which was evaluated by counting number of fecal pellets and total period of groomings. None of the swimming exercise groups exhibit a significant difference in locomotor activity, but all have shown a significant decrease in anxiety states compared to WAG/Rij control group. Conclusion: WAG/Rij rats show apparently increased locomotor activity compared to Wistars, but there is no difference between anxiety states. Short, moderate or long term exercise does not have a significant effect on locomotor activities of these animals, but calm down the anxiety states of these animals significantly.

Keywords: Absence epilepsy

\section{P-69}

Releasing flexor retinaculum in carpal tunnel syndrome patients using a variety of treatment method such as myofacial technique, mobilization of the median nerve and tendon gliding exercises and its comparison with kinesio taping techniques

Argalı $\mathrm{M}^{*}$, Ekinci $\mathrm{N}^{* *}$, Köse $\mathrm{E}^{* *}$, Altay $\mathrm{Z}^{* *}$

*Department of Physiotherapy and Rehabilitation, Faculty of Medicine, Inönü University, Malatya, Turkey; **Department of Anatomy, Faculty of Medicine, Inönü University, Malatya, Turkey
Carpal tunnel syndrome is the most common peripheral entrapment neuropathy caused by pinched median nerve in carpal tunnel. In this study, our aim is to determine the efficiency of the kinesiotaping technique that is used in physical therapy and to compare efficiency of the kinesiotaping technique with a treatment program using all three types of myofacial releasing of flexor retinaculum, mobilization of the median nerve and tendon gliding exercises. 40 volunteer female patients with CTS, divided into two groups consisted of twenty patients. The first group of the patients was received mobilization of median nerve, releasing of flexor retinaculum and tendon gliding exercises therapy for 5 days per week for 4 weeks, while the second group received the kinesiotaping technique for carpal tunnel syndrome two times per week on Mondays and Thursdays. Every patient were evaluated within the group and compared between groups according to Boston Symptom Severity Scale and Boston Functional Capacity Scale before and after the treatment program to compare the efficiencies of the treatment methods. In both groups, statistically significant reductions were observed according to Boston Symptom Severity Scale and Boston Functional Capacity Scale scores before and after the therapy. Although the difference before and after the therapy for exercise and taping group was not statistically significant according to Boston Symptom Severity Scale, a statistically significant difference was noted according to Boston Functional Capacity Scale. Positive effect on CTS symptoms was observed in both groups, but statistically significant difference was not observed between groups. Exercise group was superior to taping group, when we compare two groups according to improvement of hand functions.

Keywords: Carpal tunnel syndrome, median nerve gliding exercises, neural gliding exercises, tendon gliding exercises, kinesio taping techniques

\section{P-70}

\section{Surgical anatomy of lateral patellofemoral ligament: an anatomical study}

Ergür İ* Çapkın $\mathrm{S}^{* *}$, Zeybek $\mathrm{G}^{*}$, Koşay $\mathrm{C}^{* *}$, Kıray A*

*Department of Anatomy, Faculty of Medicine, Dokuz Eylül Universty, Izmir, Turkey; ${ }^{*}$ Department of Orthopedics and Trauma Surgery, Faculty of Medicine, Dokuz Eylül Universty, Izmir, Turkey

Patella, the largest sesamoid bone in the body has quadriceps tendon in proximal and patellar tendon in distal part. Medial patellofemoral ligament (MPFL) and lateral patellofemoral ligament (LPFL) makes the patellar attachments to femur which are both important in patellar stability. In contrast to MPFL, there are fewer studies about LPFL in the literature. LPFL is important in surgical treatment of patellar stability and patellar release. To delineate and describe the detailed anatomical specifications of LPFL. Thirty-six formaldehyde fixed knees in Dokuz Eylul University of Department of Anatomy were dissected via paramedian patellar incision. After turning the patella to lateral, the length and width at condylar attachment of 
S148 14th National Congress of Anatomy, 28 June-1 July 2012, Ankara, Turkey

LPFL were measured and attachment angle and figure in vertical and sagittal axes were inspected. Mean length of LPFL was $23.2 \mathrm{~mm}$, width at attachment to condyle was $15.6 \mathrm{~mm}$. LPFL were attached to medial part of condyle in sagittal plane in $75 \%, 53 \%$ in vertical plane and $59.4 \%$ were situated in transverse plane. LPFL were found to attach to femoral condyle in a radiating manner. Patellar fibers of LPFL were in conjunction with transverse fibers of lateral retinaculum and patellotibial band. Insertions to femoral condyle were in the middle of condyle in both planes and mostly in transverse direction. Detailed anatomical description of LPFL in this study may be important from surgical point of view in treatment of patellar instabilities and lateral patellar release.

Keywords: Lateral patellofemoral ligament, anatomical study

\section{P-71}

Morphology and morphometry of supraorbital foramen Çınar S, Ertekin T, Nisari M, Özçelik O, Ülger H

Department of Anatomy, Faculty of Medicine, Erciyes Universty, Kayseri, Turkey

Supraorbital foramen, which transmits the supraorbital nerve and vessels, is localized on medial part of supraorbital margin of frontal bone. The aim of this work was designed to determine the rate of supraorbital foramen (notch) on skulls and to find out the distances between this foramen and anthropometric points. Material and methods: This research was studied on 50 human dry skulls in the Department of Anatomy laboratory, Medical Faculty of Erciyes University. Morphometric measurements related to the supraorbital foramen (incisura) were measured using digital caliper. The mean distance between the right and left supraorbital foramen and anthropometric points were supraorbital foramen (incisura) and infraorbital foramen $(4.34 \pm 0.34 \mathrm{~cm}$ and $4.38 \pm 0.30 \mathrm{~cm})$, supraorbital foramen (incisura) and nasion $(2.74 \pm 0.54 \mathrm{~cm}$ and $2.60 \pm 0.48$ $\mathrm{cm}$ ), supraorbital foramen (incisura) and zygomaticofacial sutura $(2.97 \pm 0.33 \mathrm{~cm}$ and $3.07 \pm 0.48 \mathrm{~cm})$, supraorbital foramen (incisura) and dacryon $(2.27 \pm 0.52 \mathrm{~cm}$ and $2.20 \pm 0.33 \mathrm{~cm})$, supraorbital foramen (incisura) and glabella $(2.56 \pm 0.43 \mathrm{~cm}$ and $2.50 \pm 0.37 \mathrm{~cm}$ ), supraorbital foramen (incisura) and zygion $(6.96 \pm 0.44 \mathrm{~cm}$ and $6.96 \pm 0.38 \mathrm{~cm})$, supraorbital foramen (incisura) and superior orbital fissur $(4.71 \pm 0.34 \mathrm{~cm}$ and $4.70 \pm 0.29 \mathrm{~cm}$ ), supraorbital foramen (incisura) and optic canal $(4.26 \pm 0.27 \mathrm{~cm}$ and $4.21 \pm 0.23 \mathrm{~cm})$, supraorbital foramen (incisura) and lacrimal fossa $(2.45 \pm 0.27 \mathrm{~cm}$ and $2.53 \pm 0.51 \mathrm{~cm})$, supraorbital foramen (incisura) and infraorbital margin $(3.53 \pm$ $0.33 \mathrm{~cm}$ and $3.54 \pm 0.28 \mathrm{~cm}$ ). There were frontal incisura on 20 from 50 craniums. The localizations of supraorbital and frontal foramens are important in endoscopic, maxillofacial and orbital surgery. It is necessary to know the anatomy of this area for avoiding to damage supraorbital nerve-vessels which transmit from this foramen.

Keywords: Supraorbital foramen, morphology, morphometry

\section{P-72}

The relationship between total calvarial thickness and diploe in the elderly

Sabancioğulları $\mathrm{V}^{*}$, Salk $\dot{I}^{* *}$, Çimen $\mathrm{M}^{*}$

*Department of Anatomy, Faculty of Medicine, Cumhuriyet University, Sivas, Turkey; **Department of Radiology, Faculty of Medicine, Cumhuriyet University, Sivas, Turkey

The objective of this study is the classification of the thickness of diploe, lamina externa, lamina interna and total calvarial thickness at different points of cranium in elderly men and women. In the radiology archive, measurements were made at different points in cranial MR images of 220 (110 females, 110 males) patients, the average ages of whom were $73.23 \pm 8.40$ (age range: 61-90) and who had no disorder of the bones. Diploe thickness, lamina externa, lamina interna and total calvarial thickness were measured in eight points of the calvaria. Midfrontal point, back and front bregma, lambda, opisthocranion and euryon points were used in the measurement. The data was loaded to SPSS 16.0 program. T-test, Mann-Whitney U, Pearson correlation coefficient and Kruskal Wallis variance analysis were used in the statistical assessment. Results with a p value smaller than 0.05 were accepted as significant. Results: There was statistically significant difference in total calvarial thickness between males and females in the right euryon point only. Average total calvarial thickness at right euryon point was higher in females $(6.20 \pm 0.78 \mathrm{~mm})$ than in males $(5.96 \pm 0.68$ $\mathrm{mm})(\mathrm{p}=0.02)$. Average diploe thickness was higher in female than male except for point of bregma back $(\mathrm{p}<0.05)$. There was positive linear correlation between diploe thickness and age except for opisthocranion, right euryon and $1 \mathrm{~cm}$ inferior to lambda. These results related to diploe thickness and cranium thickness may be leading in the determination of sex and age; surgical interventions to the cranium and bone graft choice and may increase the reliability of the operation.

Keywords: Diploe thickness, anthropologic points, lamina interna, lamina externa, MRI

\section{P-73}

\section{Mesencephalic tectum dimensions according to age groups in the MRI}

$\underline{\text { Sabancioğulları } \mathrm{V}^{*}}$, Salk $\mathrm{I}^{* *}$, Balaban $\mathrm{H}^{* * *}$, Öztoprak $\dot{I}^{* *}$, Kelkit $\mathrm{S}^{* * * *}$, Çimen $\mathrm{M}^{*}$

*Department of Anatomy, Faculty of Medicine, Cumhuriyet University, Sivas, Turkey; **Department of Radiology, Faculty of Medicine,

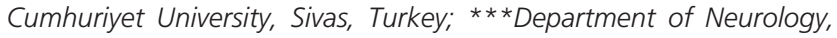

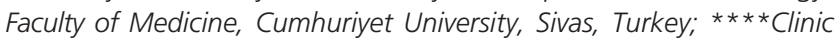
of Radiology, Sivas Numune Hospital, Sivas, Turkey

Some of the pathologies located on the mesencephalic tectum may lead to enlargement or becoming smaller of the colliculi without actually inducing any signal change on MR images. Therefore, we analyzed the normal tectum dimensions and their change according to age by classifying them according to 
age groups in a large sampling group. Tectum length and colliculus diameters were measured on T1 midsagittal and axial cranial MR images in the radiology archive belonging to 532 (344 women, 188 men) patients who were $37.36 \pm 21.49$ (4-91) years old on average and did not have any disorders that could affect mesensephalic tectum. The obtained data was loaded on SPSS 16.0 program. T-test, Mann-Whitney U, Kruskal Wallis variance analysis and Pearson correlation coefficient were used in statistical assessment. Results whose $\mathrm{p}$ value was lower than 0.05 was regarded as meaningful. Though there was a positive linear correlation between tectum length and age, there was a negative correlation between the anteroposterior diameter of colliculus superior and colliculus inferior and age $(\mathrm{p}<0.01)$. While tectum length (M3) increases with age, anteroposterior diameter of colliculus superior and inferior (M1 and M2) decreased $(\mathrm{p}<0.01)$. Colliculi were larger and tectum was longer in men. Despite the fact that there was no difference in size between right and left superior colliculi, left colliculus inferior was larger than the right one. In addition to the fact that normal mesencephalic tectum dimensions which we classified according to age groups give us information about the brain development of individuals, they may be beneficial for the detection of pathologies that affect the shape and size of colliculi without changing signal intensity on MR images and facilitate treatment. "This work is supported by the Scientific Research Project Fund of Cumhuriyet University under the project number T-474."

Keywords: Mesencephalic tectum; colliculus superior; colliculus inferior; MRI; morphometry.

\section{P-74}

\section{Watershed area after vascular injury of the sciatic nerve: effect of tacrolimus on size of watershed areas}

Hizay $A^{*}$, Demirel BM*, Gökhan $G^{* *}$, Sarıkcıŏglu L*, Demir $\mathrm{N}^{* * *}$

*Department of Anatomy, Faculty of Medicine, Akdeniz University, Antalya, Turkey; ${ }^{*}$ Department of Pathology, Faculty of Medicine, Akdeniz University, Antalya, Turkey; ${ }^{* *}$ Department of Histology and Embryology, Akdeniz University, Antalya, Turkey

Tacrolimus is an immunosuppressant drug which is used to prevent rejection after organ transplantation. Although there are several studies on neuroprotective effect of Tacrolimus on brain ischemia, few reports on effects of Tacrolimus after peripheral nerve ischemia have been reported. In the present study, we examined the size of watershed area after stripping of the epineural vessels and studied the effect of Tacrolimus on reduction of the size of watershed area. Forty-eight adult female rats were used and randomly divided into four groups as control, sham, Tacrolimus-treated and vehicle-treated. In Tacrolimustreated and vehicle-treated groups epineural vessels around the sciatic nerve (vasa nervorum) were stripped. Additionally, Tacrolimus-treated group were received subcutaneous injection of $5 \mathrm{mg} / \mathrm{kg}$ Tacrolimus. Percent of watershed area (100xtotal watershed areas / total nerve area) after stripping and Tacrolimus treatment was calculated. Numerous damaged myelin residues were observed in the vehicle and Tacrolimustreated groups. The amount of myelin debris was higher in the vehicle-treated group than those in the Tacrolimus-treated group. Comparison of the total nerve areas revealed that there was no statistical significance between all groups of the study. In the literature it has not, to our knowledge, been reported the potential of the tacrolimus treatment on reduction of the percent of watershed area in sciatic nerve after ischemic or devascularization injury. We think that this study will be helpful for neuroscientist who dealing with peripheral nerve injury.

Keywords: Epineural vessels, tacrolimus, sciatic nerve, vasa nervorum

\section{P-75}

\section{A case with bilateral variations of the branches of the internal iliac artery}

Gilan IY*, Koç $T^{*}$, Aktekin $M^{* *}$

*Institute of Health Sciences, Mersin University, Mersin, Turkey; ${ }^{*}$ Department of Anatomy, Faculty of Medicine, Mersin University, Mersin, Turkey

It is aimed to discuss the branching pattern of the internal iliac artery based on a case which had unusual origins of the obturator and iliolumbar arteries bilaterally. Case: During the pelvis dissection of an 81 year old male cadaver, it was found that the iliolumbar and obturator arteries on both sides originated from different levels of the internal iliac artery. On the right side; internal iliac artery gave its iliolumbar branch posteromedially $34.63 \mathrm{~mm}$ distal to its origin, then diverged into anterior and posterior trunks. Posterior trunk gave the lateral sacral artery $1.35 \mathrm{~mm}$ and the obturator artery $23.60 \mathrm{~mm}$ from its diverging point, and reached to the greater sciatic foramen as the superior gluteal artery. The anterior trunk gave the superior vesical branch $1 \mathrm{~mm}$ after diverging from the internal iliac artery. No other variation was observed regarding the branches of the anterior trunk. On the left side, similar to the right side, the internal iliac artery gave the iliolumbar branch posteromedially $18.87 \mathrm{~mm}$ distal to its origin. $20.87 \mathrm{~mm}$ distal to this point, it gave the superior vesical branch and after $9.25 \mathrm{~mm}$ it diverged into the anterior and posterior trunks. The posterior trunk gave the lateral sacral branch $7 \mathrm{~mm}$ and the obturator artery $22.40 \mathrm{~mm}$ after its origin and continued as the superior gluteal artery towards the greater sciatic foramen. No other variation was observed regarding the other branches of the anterior trunk on this side. A bilateral pattern that the obturator arteries diverge from the posterior trunk and the iliolumbar arteries diverge from the internal iliac arteries makes the present case exceptional. All these variations should be considered for a safe surgical intervention of the pelvic region.

Keywords: Obturator artery, iliolumbar artery, internal iliac artery, pelvis, variation 
S150 14th National Congress of Anatomy, 28 June-1 July 2012, Ankara, Turkey

\section{P-76}

\section{A variation of the lateral circumflex femoral artery: case report}

Soyluoğlu Aİ, Kahraman G, Üzel M, Tanyeli E

Department of Anatomy, Cerrahpaşa Medical Faculty, Istanbul University, Istanbul, Turkey

During the educational dissections in 2011-2012 term, a variation of the lateral circumflex femoral artery (LCFA) was found at the right lower extremity of a 65 -year-old male cadaver. The ascending branch of the LCFA (AsBr) branched from the femoral artery (FA) proximal to the deep femoral artery's (DFA) branching point. The distance between the inguinal ligament (IL) and the origin of the AsBr was $2.7 \mathrm{~cm}$. The descending branch of the LCFA (DeBr) branched from the FA as well, but distal to the DFA's branching point. The distance between the inguinal ligament (IL) and the origin of the $\mathrm{DeBr}$ was $10.8 \mathrm{~cm}$. Discussion: The incidence of such a variation is between $0.9-1.5 \%$ in the literature. Both branches of the LCFA have important clinical implications. The AsBr can be used as a supply for vascularized iliac transplantation, while the $\mathrm{DeBr}$ can act as collateral in obstructed FA, and can be used in coronary artery bypass grafting. The branching pattern and the distances of the AsBr and $\mathrm{DeBr}$ are of importance in surgical or angiographic interventions, and variations at the region should be remembered during procedures

Keywords: Anatomy, variation, femoral artery, deep femoral artery, lateral circumflex femoral artery

\section{P-77}

\section{Evaluation of perception of time and color in children} and adults

Akın FA*, Akın $M^{*}$, Ensari A*, Gazioğlu $C^{*}$, Tokgönül S*, Elhan $\mathrm{AH}^{* *}$, Çiçek $\mathrm{M}^{* * *}$

*Medical Student, Faculty of Medicine, Ankara University, Ankara, Turkey; **Department of Biostatistics, Faculty of Medicine, Ankara University, Ankara, Turkey; ***Department of Physiology, Faculty of Medicine, Ankara University Ankara, Turkey
The aim of our study was to compare time and colour perception among adults and children and especially to reveal the age dependent change of time perception. Two groups of children (n:30; age:7-12, \%50 male, \%50 female) and adult group (n:30, 20,25, \%60 male, \%40 female) were selected randomly. The participants were selected among the students of Karataş Elementary School for the children group and the students of AUTF for adult groups. Research has been conducted between the dates of February-April 2012. Participants' compliancy of the criteria of the inclusion to the research is determined. Time and color perception is measured by Foreperiod Test which is designed by using MATLAB software. Percentage of correct responses and reaction times of each participant for the two tasks were obtained. Data was analyzed in SPSS programme using Paired T Test and Two -Way ANOVA. Percentage of correct response and reaction time is analysed with two-way ANOVA separately. For percentage of correct response; task effect $(\mathrm{F}=64.2, \mathrm{p}<0,001)$ and group effect were significant $(\mathrm{F}=91.8, \mathrm{p}<0.001)$. Task-group interaction was significant $(\mathrm{F}=4.5, \mathrm{p}<0.05)$. For reaction time; group effects was significant $(\mathrm{F}=21.8 \mathrm{p}<0.001)$. Task effect and interaction of task-group were not significant. Reaction times for time and colour perception tasks were not different from each other both in adult groups and children groups $(\mathrm{p}>0.05)$. However, percentage of correct responses for the two tasks were different in both groups (for children, $\mathrm{t}=5.5, \mathrm{p}<0.001$; for adults $\mathrm{t}=8.3, \mathrm{p}<0.001$ ). Participants' time perception performances were lower than their colour perception. The difference of performances was distinct in children than adults. Children group displayed lower performance in both task than adults. These results indicate that time perception in human being has a slower development than colour perception. The future research about the change of perception of time according to age can provide additional information and this information might be useful to understand the reason of illnesses such as major depression and promote educational programmes.

Keywords: Time perception, color perception, behavior tests, developmental difference, and performance 